\title{
Noise-robust range alignment method for inverse synthetic aperture radar based on aperture segmentation and average range profile correlation
}

\author{
Yue Lu' ${ }^{1}$, Jian Yang ${ }^{2 *}$, Yue Zhang ${ }^{1}$ and Shiyou $X^{3}$
}

\begin{abstract}
Range alignment is an essential procedure in the translation motion compensation of inverse synthetic aperture radar imaging. Global optimization or maximum-correlation-based algorithms have been used to realize range alignment. However, it is still challenging to achieve range alignment in low signal-to-noise ratio scenarios, which are common in inverse synthetic aperture radar imaging. In this paper, a novel anti-noise range alignment approach is proposed. In this new method, the target motion is modeled as a uniformly accelerated motion during a short sub-aperture time. Minimum entropy optimization is implemented to estimate the motion parameters in each sub-aperture. These estimated parameters can be used to align the profiles of the current sub-aperture. Once the range profiles of each sub-aperture are aligned, the non-coherent accumulation gain is obtained by averaging all profiles in each sub-aperture, which can be used as valuable information. The accumulation and correlation method is applied to align the average range profiles of each sub-aperture because the former step focuses mainly on alignment within the sub-apertures. Experimental results based on simulated and real measured data demonstrate the effectiveness of the proposed algorithm in low signal-to-noise ratio scenarios.
\end{abstract}

Keywords: Aperture segmentation, Entropy minimization, Inverse synthetic aperture radar, Range alignment

\section{Introduction}

Inverse synthetic aperture radar (ISAR), which is used to obtain images of non-cooperative and moving targets, has been widely applied in many civil and military domains in the last few decades [1-5]. ISAR achieves high resolutions in both the range and azimuth directions by exploiting the wideband characteristics and angular diversity during the coherent processing interval. During the process of imaging, a target's movement can be divided into two parts: translational and rotational motion. It is well known that only the rotational motion contributes to imaging, while translational motion can cause blurring of the ISAR images and therefore must be compensated for. Range

\footnotetext{
*Correspondence: yangjian@nudt.edu.cn

${ }^{2}$ Rocket Force University of Engineering, Xi'an 710025, China

Full list of author information is available at the end of the article
}

alignment and autofocusing constitute the basic workflow of translational motion compensation. The range alignment procedure is the prerequisite for the subsequent fine-tuned phase compensation, which ensures that the amount of energy of the same scatters are in the same range bins. Well-aligned range profiles are the foundation of a focused ISAR image [6-9]. This article focuses mainly on a novel and effective method for range alignment.

The traditional range alignment methods can be sorted into three categories. The first is maximum-correlationbased methods, which maximize the correlation function between adjacent profiles or between the current profile and a template one. A representative one was discussed in detail in [1]. To improve the performance of these methods in low SNR scenarios, [10] proposed that the average of aligned profiles can be used as a reference. Xue 
et al. [11] demonstrated a high-order symmetric accumulated cross-correlation method (HSACM) to achieve a high real-time computation speed. In extremely low SNR scenarios, however, these methods can be impacted by noise, and misalignment errors accumulate during the coherent processing interval (CPI). The second category of methods is based on the dominant scatters of targets $[12,13]$. Although these methods have high computational efficiency, they are sensitive to angular glint and noise, and they have difficulty in tracking strong scatters in real situations. The third category of methods is optimization-based and uses several global metrics, such as entropy $([14,15])$ and contrast $([16,17])$, as references to iteratively compensate for misalignment. However, as mentioned in [18], the synthetic profiles in the global methods are equal to the non-coherent accumulation, and under a low SNR, the SNR gain from the non-coherent integrant is not sufficient to overcome the strong noise, and as a result, the metrics cannot fully indicate the quality of the range alignment. In short, the current range alignment methods suffer performance reductions in low SNR situations, and it is still challenging to propose an anti-noise range alignment method.

By referring to [19] and [20], it is likely that ISAR signals encounter interference from strong noises because of the long observation distance and low transmitting energy. For example, when the target of interest is a high-orbit satellite, the SNR of echoes can be very low. It has been concluded that if the required SNR decreases by $1 \mathrm{~dB}$, the surveillance range will increase by $8 \%$. Therefore, it is essential to implement algorithms with high robustness under low SNRs. Methods such as those by [18] and [19] model the total translation motion as a high-order polynomial and use coordinate descent or a particle swarm algorithm to carry out the optimization. According to [21] and [22], the plain coordinate descent used in [18] can easily be trapped in a local optimum when the cost function is non-convex. The particle swarm algorithm is heuristic, which means a relatively complex parameter setting scheme is required. In addition, the above algorithms jointly solve range alignment and phase compensation, but none of them is specifically aimed at range alignment. Furthermore, joint compensation demands higher accuracy (range, 1/4 1/8 range unit; phase, wavelength/8) than that of the separated methods, and joint compensation requires a high-order model, which represents a high computational cost.

To improve the performance of the range alignment methods in low SNR scenarios and overcome the shortcomings of the above algorithms, the proposed method is designed with the following three steps. First, the target motion information is considered. In a relatively short observation time, it is reasonable to model the target motion as uniform acceleration. However, in a real situation, the CPI is always long. Therefore, we split the full aperture into several sub-apertures to achieve a short observation time. Extra information is of benefit to suppress strong noise. In each sub-aperture, the minimum entropy principle is implemented to estimate the motion parameters and obtain the envelope deviations of all profiles in the current sub-aperture. As an improvement to [18], the coordinate descent algorithm (CDA) with proximal point updating is used to obtain better convergent properties. Second, after optimization and compensation

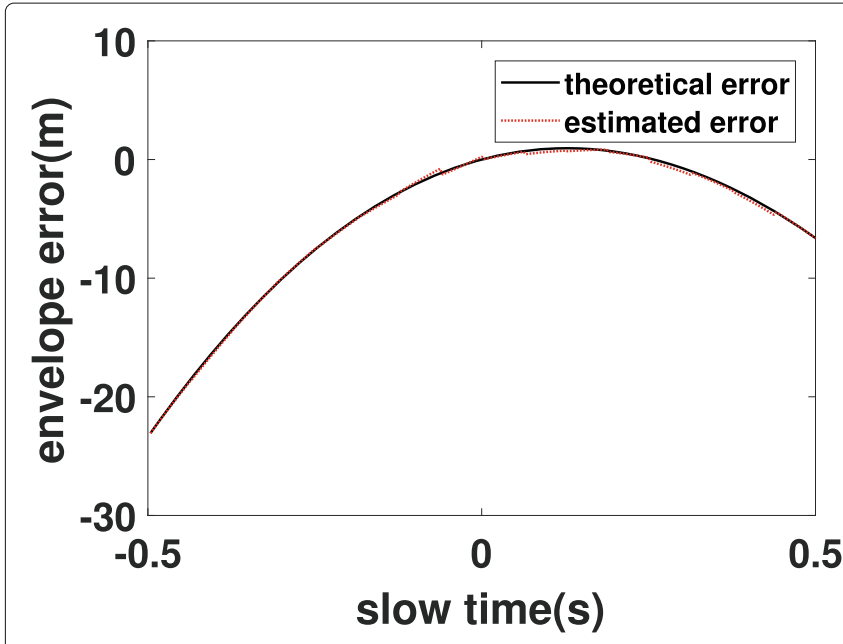

(a)

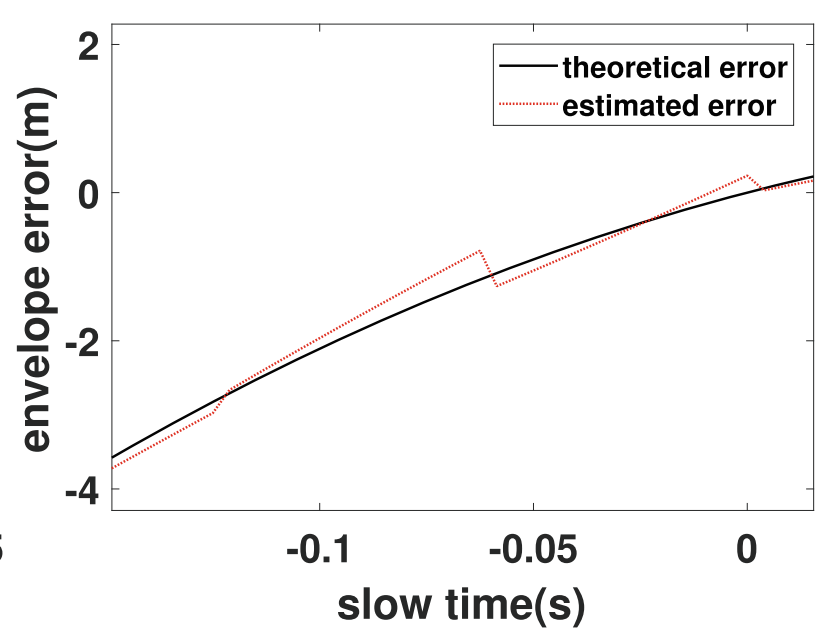

(b)

Fig. 1 Estimated error curves without fitting. a Theoretical and estimated error curves and $\mathbf{b}$ enlarged version of $\mathbf{a}$ 
in each sub-aperture, non-coherent accumulation gain can be realized through the average of the profiles in the sub-aperture, and we then use a maximum-correlationbased algorithm to align the average range profiles of every sub-aperture and obtain the envelope deviation between every two sub-apertures. This step is necessary because optimization in each sub-aperture mainly focuses on the alignment of the current sub-aperture. After the former two steps, the envelope deviations of all profiles in one sub-aperture and the deviations of the average range profiles of every sub-aperture, i.e., the error within and between sub-apertures, are obtained. Finally, locally weighted regression is applied to fit the total envelope deviations to eliminate the step change between every two sub-apertures. In general, the reason that the proposed method has enhanced performance in low SNR scenarios is that the target motion information and global optimization are used, and in addition, the non-coherent accumulation gain is adopted.

The main contributions of this article can be summarized as follows:

1) A novel range alignment approach is proposed with consideration of the target motion, and the global optimization and maximum-correlation-based methods are combined to achieve high performance in low SNR scenarios.

2) The CDA with a proximal update scheme is used to achieve stability. Furthermore, in each iteration of the CDA, the Levenberg-Marquardt (LM) method is implemented as the solver. The LM method, which blends the advantages of the gradient descent and Newton's method, can achieve a better robustness and a faster rate of convergence.

The remainder of this paper is organized as follows. In Section 2, the signal model is introduced, and related works, such as the maximum-correlation-based algorithm (MCA) and minimization of the entropy of the average range profile (MEARP) [7], are briefly summarized for comparison. In Section 3, the three-step procedure of the proposed algorithm is discussed in detail. Section 4 presents experimental results based on simulated and real measured data to verify the effectiveness of the proposed algorithm. Some conclusions are presented in Section 5.

\section{Signal model and related works}

In this section, the models of transmitted and received signals obtained using the dechirping technique are briefly deduced, and the formula of envelope deviation

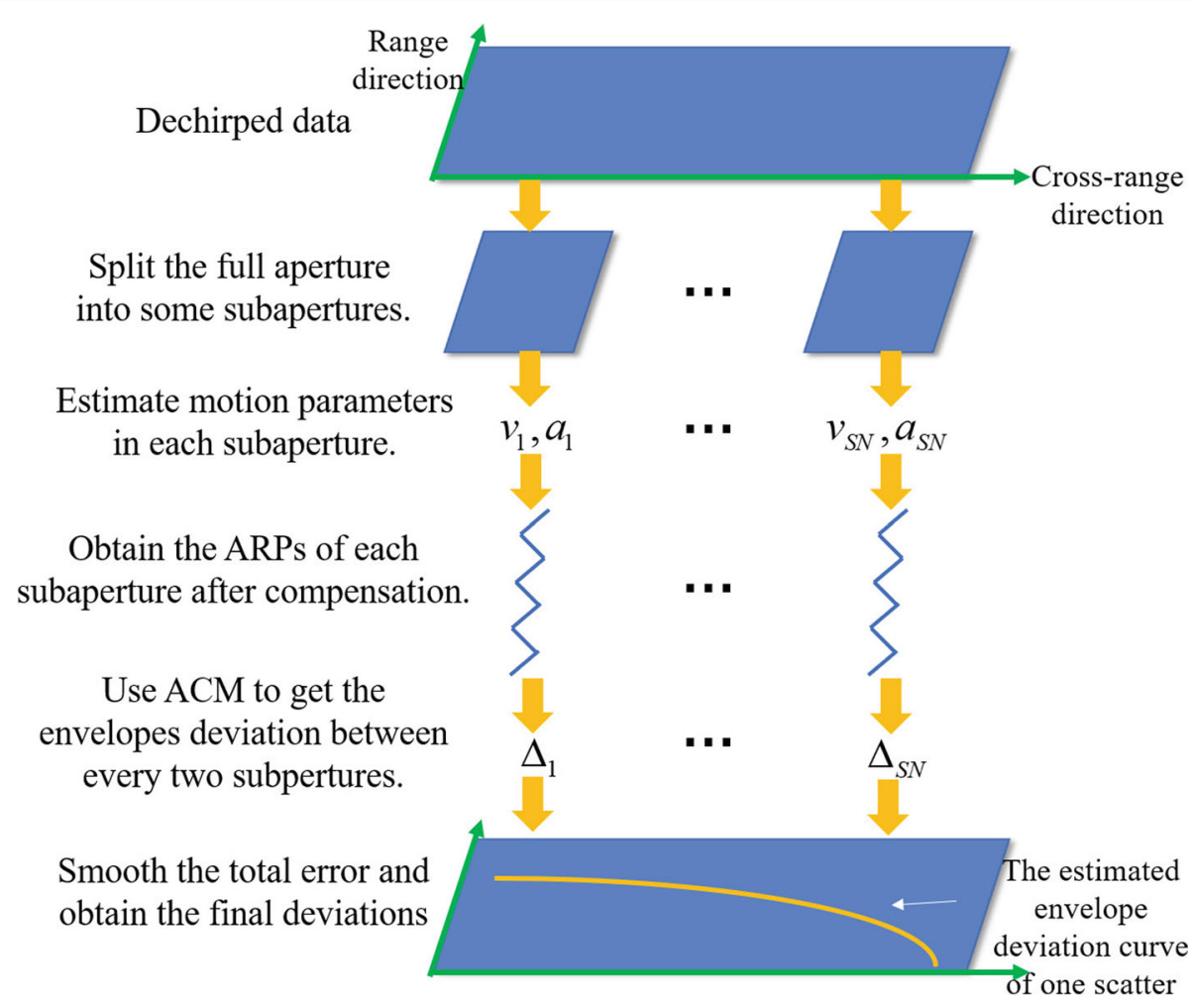

Fig. 2 Framework of the proposed algorithm 
compensation is demonstrated. For comparison, a barebones introduction to the related works, including the MCA [1] and MEARP [7], is presented.

\subsection{Signal model}

In ISAR imaging, the linear frequency modulation (LFM) signal is often transmitted, which can be expressed as

$$
s(\hat{t})=\operatorname{rect}\left(\frac{\hat{t}}{T_{P}}\right) \cdot \exp \left[j 2 \pi\left(f_{c} \hat{t}+\frac{1}{2} \gamma \hat{t}^{2}\right)\right]
$$

where $\operatorname{rect}(\cdot)$ is the rectangular function $\left(\operatorname{rect}(\tau)=\left\{\begin{array}{l}1,|\tau| \leq \frac{1}{2} \\ 0,|\tau|>\frac{1}{2}\end{array}\right) . T_{p}, f_{c}, \gamma\right.$, and $\hat{t}$ represent the pulse duration, carrier frequency, chirp rate, and fast time, respectively.

After applying the well-established dechirping technique, including residual video phase (RVP) term elimination, and the fast Fourier transform (FFT) operations to the fast time, high-resolution range profiles (HRRPs) can be obtained:

$$
\begin{gathered}
S\left(f, t_{s}\right)=A \cdot T_{P} \cdot \sin c\left(T_{P}\left(f+2 \frac{\gamma}{c} \Delta R\right)\right) \\
\cdot \exp \left(-j \frac{4 \pi f_{c}}{c} \Delta R\right)
\end{gathered}
$$

where $A, f$, and $\Delta R$ denote the amplitude modulation, frequency variable, and radial range difference between the scatter and reference point, respectively. $t_{s}$ is the slow time with $0 \leq t_{s} \leq T_{a}$, where $T_{a}$ is the CPI [18], and the variation in $t_{s}$ is reflected by $\Delta R$ :

$$
\begin{aligned}
\Delta R & =R_{0}-R_{\text {ref }} \\
& \approx d \cdot \sin \left(\Omega t_{s}\right)
\end{aligned}
$$

where $R_{0}$ is the radial distance between the scatter and the radar, $R_{\text {ref }}$ is that between the reference point and the radar, $d$ is the distance between the scatter and the reference point, and $\Omega$ represents the angular velocity of the target.

The range misalignment, due to the translation motion of the target, can be reflected by the envelopes of the HRRPs under the dechirping model. The peak of the sinc function in (2), which is located at $f=-2 \gamma \Delta R / c$, denotes the range information of the scatter. However, because of the variation in $\Delta R$ during the $C P I$, the peaks exist in different range bins among all sweeps of the radar, resulting in the defocusing of the scatter energy in the final ISAR image.

To achieve range alignment, the misaligned envelopes of the HRRPs should be shifted along the direction of fast

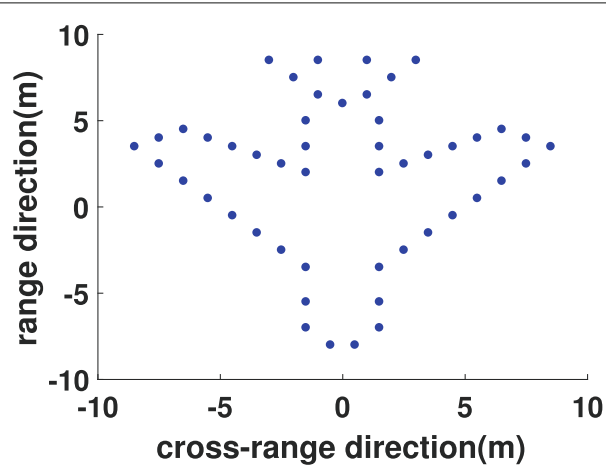

(a)

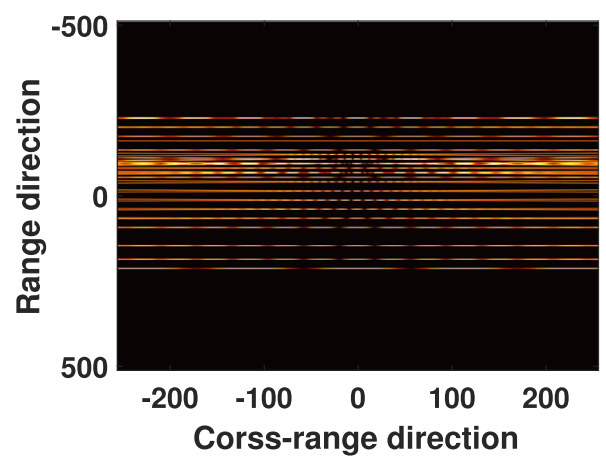

(c)

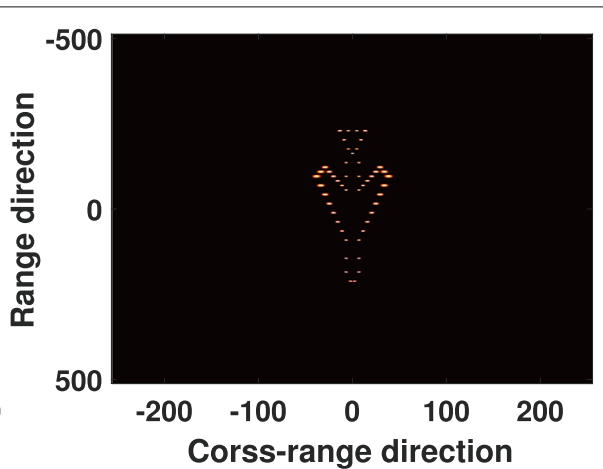

(b)

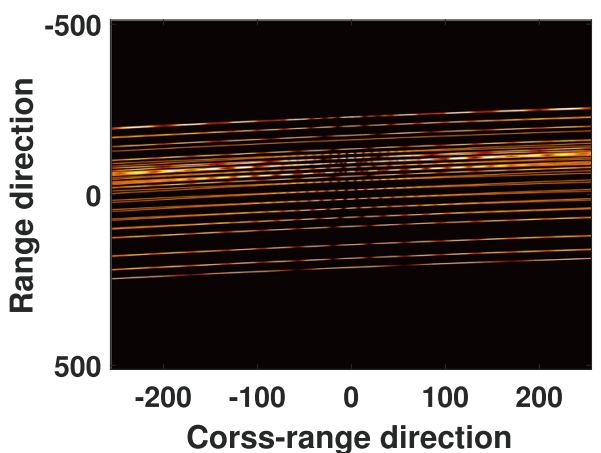

(d)

Fig. 3 The plane model. a The simulated plane model, $\mathbf{b}$ the original ISAR image, $\mathbf{c}$ the original range profiles, and $\mathbf{d}$ the contaminated range profiles 
time. According to the shift property of the Fourier transform, alignment can be accomplished in the time domain as follows:

$$
\tilde{s}\left(t_{s}, \hat{t}\right)=s\left(t_{s}, \hat{t}\right) \cdot \exp \left[-j 2 \pi \hat{t} \Phi\left(t_{s}\right)\right]
$$

where $\tilde{s}\left(t_{s}, \hat{t}\right)$ is the compensated signal echo and $\Phi\left(t_{s}\right)$ represents the envelope deviations of different sweeps.

\subsubsection{Related works}

The two main approaches to achieving range alignment are the MCA and MEARP. In this subsection, both are briefly introduced.

One of the widely used maximum-correlation-based algorithms is the accumulation and correlation method (ACM), which uses the average of already aligned envelopes as the template (reference envelope) and maximizes the correlation function between the template and the next unaligned envelope. Its formula is as follows:

$$
\tau_{s}=\arg \max _{\Delta_{\tau}} R\left(x_{i}\left(\Delta_{\tau}\right), \sum_{m=1}^{Q} x_{m}\right)
$$

where $x_{i}$ and $x_{m}(m=1,2, \ldots, Q)$ represent the $i$ th unaligned and first $Q$ aligned envelopes, respectively. $R(\cdot)$ denotes the correlation function, which is maximized with respect to $\Delta_{\tau}$, i.e., the shift value of the envelope. $\tau_{s}$ is the final result used to calibrate the current envelope. The approaches for averaging the already aligned envelopes in (5) can be varied for the sake of accuracy and stability [10, 23].

For the MEARP, it is iterated with the entropy minimization principle as follows [7]:

$$
\begin{aligned}
& \Delta_{\tau}^{(k)}(m)= \Delta_{\tau}^{(k-1)}(m) \\
&+\arg \left\{\max _{\tau} R\left(p\left(\tau+\Delta_{\tau}^{(k-1)}(m), m\right), \tilde{p}_{\text {ave }}^{(k-1)}(\tau)\right)\right\} \\
& m=0, \ldots, M-1
\end{aligned}
$$

and

$$
\tilde{p}_{\text {ave }}^{(k-1)}=\ln p_{\text {ave }}^{(k-1)}=\ln \left\{\sum_{m=0}^{M-1} p\left(\tau+\Delta_{\tau}^{(k-1)}(m), m\right)\right\}
$$

In (6) and (7), $r$ and $m$ represent the radial range and pulse index, respectively. $k$ denotes the iteration number, and $p(r, m)$ is the envelope of the range-compressed ISAR signal. The other mathematical symbols are the same as those in (5).

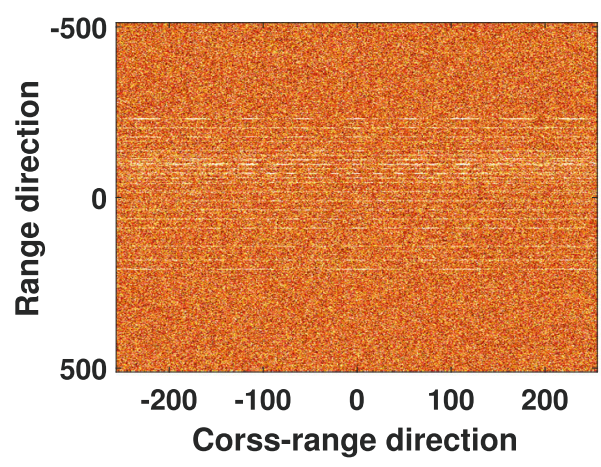

(a)

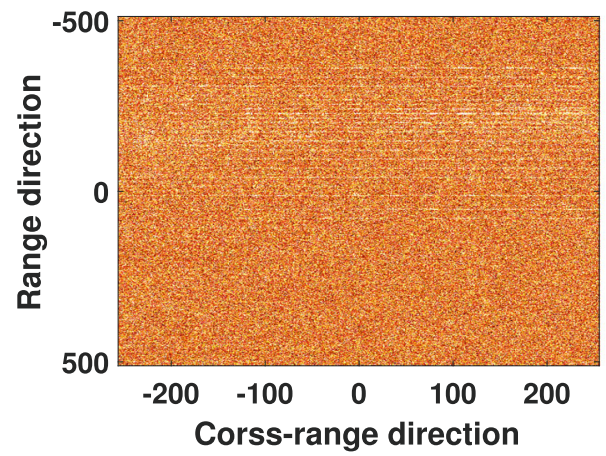

(c)

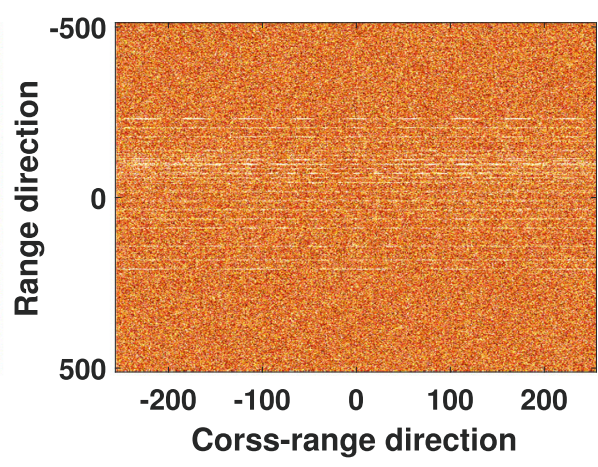

(b)

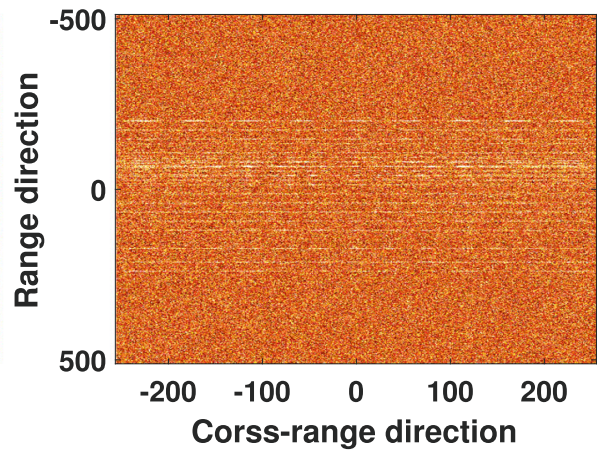

(d)

Fig. 4 Alignment results of the different methods under a - $10 \mathrm{~dB}$ SNR. a The range profiles without envelope deviation, $\mathbf{b}$ the alignment result of the proposed algorithm, $\mathbf{c}$ the alignment result of the ACM, and $\mathbf{d}$ the alignment result of the MEARP 
The two algorithms above are representative of the maximum-correlation-based and global-optimizationcriterion-based methods, respectively. They are used for comparison with the proposed algorithm.

\section{Methods}

In this section, the workflow of the proposed range alignment method is discussed in detail. In general, as mentioned in Section 1, this novel algorithm contains three steps:

1 Split the full aperture and estimate the target motion parameters in each sub-aperture based on the CDA and the Levenberg-Marquardt (LM) optimization method

2 Align the envelopes of the average range profiles (ARPs) of every sub-aperture using the MCA

3 Smooth the estimated deviations by means of locally weighted regression (LOESS)

\subsection{Estimating the motion parameters in the sub-apertures}

Traditional range alignment methods often focus on the similarity among range profiles without fully considering the target motion information. As a result, these algorithms are vulnerable in low signal-to-noise ratio (SNR) scenarios.

In a relatively short observation time, the target translation motion can be regarded as stable movement, i.e., uniformly accelerated motion; thus, it is rational to model the envelope shift as a second-order polynomial with respect to the slow time. With the adoption of the motion information, the algorithm robustness under a low SNR can be considerably improved. However, in real ISAR imaging, hundreds or even thousands of echoes are accumulated, which leads to a long observation time. To use the motion information, we split the full aperture into a certain number of sub-apertures to obtain a short slow-time span. In each sub-aperture, optimization based on the minimum entropy principle is performed to estimate the velocity and acceleration of the target, which is discussed at length in the following.

Assume every $M$ consecutive echoes of the full aperture are viewed as a sub-aperture and that the echo in the $k$ th sub-aperture is $x_{k}\left(t_{m}, \hat{t}\right)$, where $t_{m}$ is the slow time and $t_{n}$ is the fast time (discrete). As mentioned above, the envelope shift in one sub-aperture can be modeled as a second-order polynomial in $t_{m}$ with two unknown

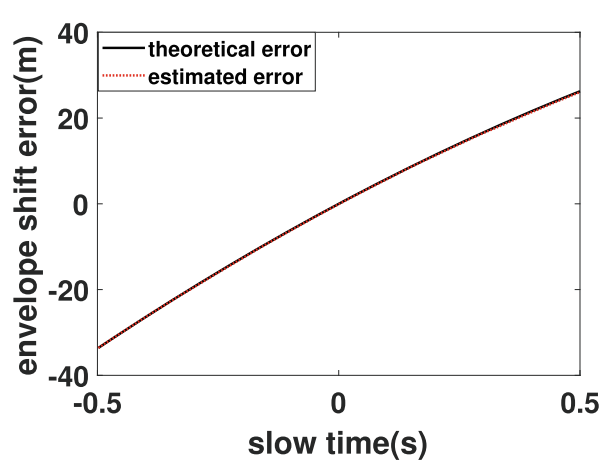

(a)

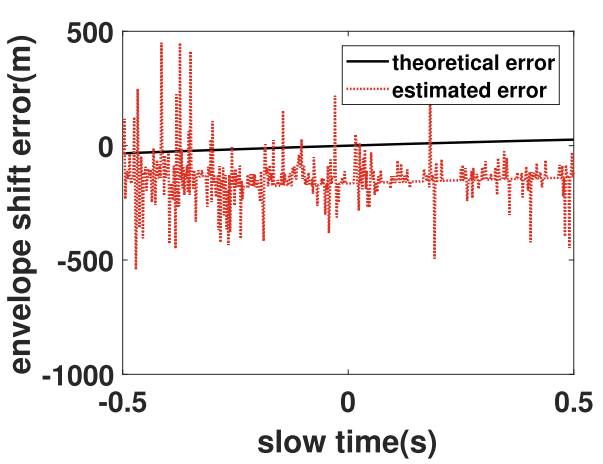

(b)

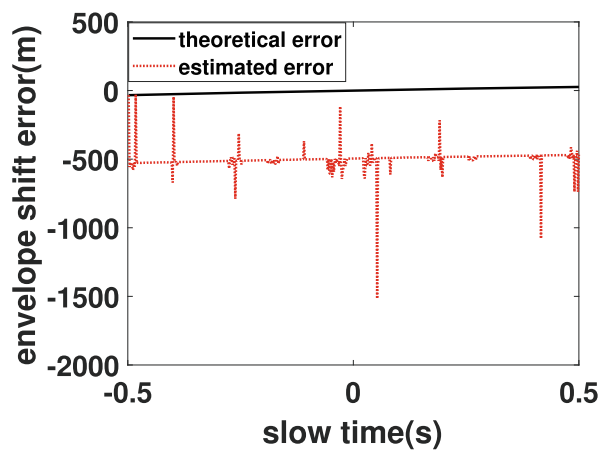

(c)

Fig. 5 Estimated error curves of the three methods under a - $10 \mathrm{~dB}$ SNR. a The estimated error curve of the proposed algorithm, $\mathbf{b}$ the estimated error curve of the ACM, and $\mathbf{c}$ the estimated error curve of the MEARP 


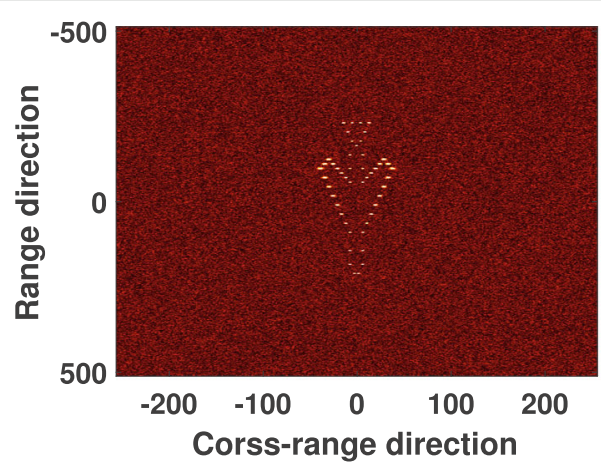

(a)

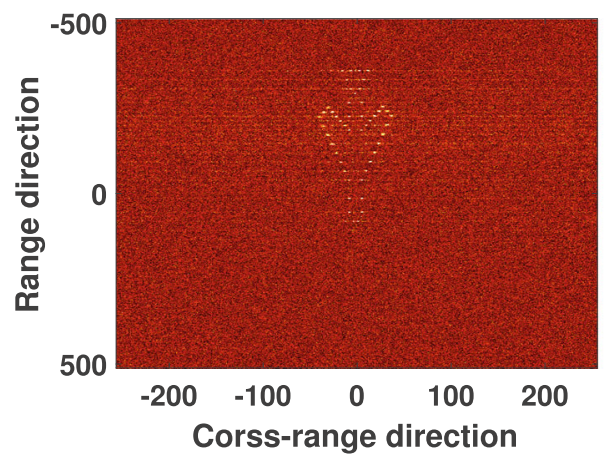

(c)

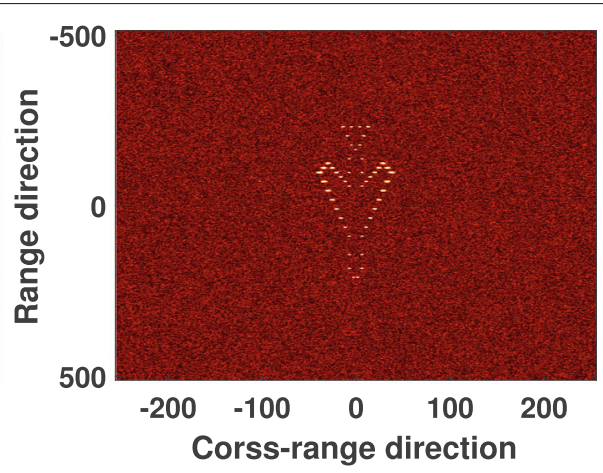

(b)

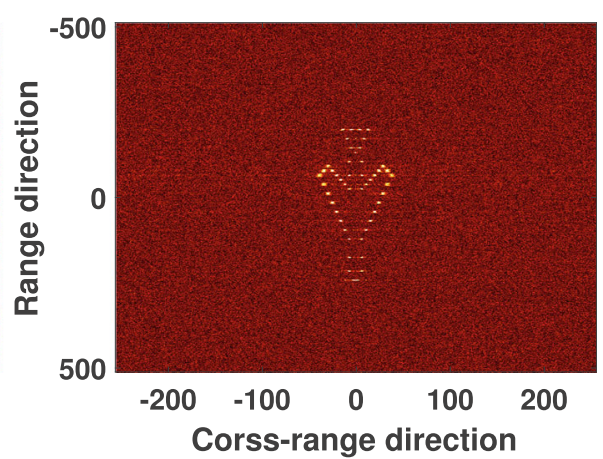

(d)

Fig. 6 Imaging results of the different methods under a - $10 \mathrm{~dB}$ SNR. a The ideal ISAR image, $\mathbf{b}$ the imaging result of the proposed algorithm, $\mathbf{c}$ the imaging result of the ACM, and $\mathbf{d}$ the imaging result of the MEARP

parameters, i.e., acceleration $a$ and velocity $v$ :

$$
\Phi\left(t_{m}\right)=v t_{m}+a t_{m}^{2}
$$

where $\Phi\left(t_{m}\right)$ represents the envelope shift error.

As (4) indicates, once the optimal values of the unknown parameters are obtained, modulation in the time domain can be carried out to compensate for the range shift:

$$
\tilde{x}_{k}\left(t_{m}, t_{n}\right)=x_{k}\left(t_{m}, t_{n}\right) \cdot \exp \left[-j 2 \pi t_{n}\left(v t_{m}+a t_{m}{ }^{2}\right)\right]
$$

where $\tilde{x}_{k}$ denotes the compensated echo.

To estimate $a$ and $v$, the minimum entropy method is introduced. By referring to [24], compared with contrastbased methods, this method can attain a good compromise among all kinds of scatters contained in the echo and result in a globally high-quality image. The entropy of the ARP of the current sub-aperture is chosen as the metric, and the unknown parameters are estimated by minimizing the entropy.

The HRRP of one sub-aperture is denoted by

$$
H R R P=f f t\left\{\tilde{x}_{k}\left(t_{m}, t_{n}\right)\right\}
$$

where $f f t\{\cdot\}$ represents the FFT operation on $t_{n}$.
Based on (9) and (10), the ARP of the sub-aperture can be expressed as:

$$
\begin{aligned}
A R P & =\frac{1}{M} \sum_{m=0}^{M-1}\left|f f t\left\{\tilde{x}_{k}\left(t_{m}, t_{n}\right)\right\}\right| \\
& =\frac{1}{M} \sum_{m=0}^{M-1}\left|f f t\left\{x_{k}\left(t_{m}, t_{n}\right) \cdot \exp \left[-j 2 \pi t_{n}\left(v t_{m}+a t_{m}^{2}\right)\right]\right\}\right| \\
& \triangleq \frac{1}{M} \sum_{m=0}^{M-1}\left|f_{k}(v, a)\right|
\end{aligned}
$$

where $f_{k}(v, a)=f f t\left\{x_{k}\left(t_{m}, t_{n}\right) \cdot \exp \left[-j 2 \pi \hat{t}\left(v t_{m}+a t_{m}^{2}\right)\right]\right\}$ and $m$ is the index of each echo in the sub-aperture. Equation (11) is apparently a one-dimensional real

Table 1 Comparison of simulated imaging results under a $-10 \mathrm{~dB}$ SNR

\begin{tabular}{lllll}
\hline & Original image & Proposed method & ACM & MEARP \\
\hline Entropy & 12.52841 & 12.52842 & 12.68372 & 12.60427 \\
Contrast & 0.58952 & 0.58952 & 0.55225 & 0.57155 \\
\hline
\end{tabular}


function with a length equal to the number of fast time sampling points $N$.

According to [18], the entropy of the ARP can be written as

$$
E(A R P)=-\frac{1}{S_{\text {arp }}} \sum_{n=0}^{N-1}|A R P|^{2} \ln |A R P|^{2}+\ln S_{\text {arp }}
$$

where $n$ is the index of the fast-time sampling points and $S_{\text {arp }}$ is the intensity of the ARP, namely,

$$
S_{\text {arp }}=\sum_{n=0}^{N-1}|A R P|^{2}
$$

Combining (11) and (12), it can be seen that the entropy $E$ is a function of the unknown parameters $a$ and $v$. Therefore, the problem of estimating these parameters can be abstracted to the following form:

$$
\langle\hat{a}, \hat{v}\rangle=\underset{a, v}{\arg \min } E(a, v)
$$

where $\hat{a}$ and $\hat{v}$ are the estimated values that minimize the entropy.

Equation (14) is a two-dimensional optimization. In the proposed algorithm, the CDA is implemented as the optimization solver, which is an iterative method with outer and inner iterations. The inner ones, with the same number of unknown parameters, are accomplished by minimizing the objective along a certain dimension while fixing the remaining components of the vector of the parameters at their current values. The outer one is not to be terminated until the criteria on the tolerance of the change in the cost function or the preset maximum loop times are met [21].

According to [25], by using a proximal point update technique, the CDA can achieve better robustness in solving one-dimensional sub-problems. Suppose the vector of the unknown parameters is $\theta$ and that the CDA procedure is in the $p$ th outer loop and implemented to update the $i_{p}$ th parameter. The CDA updating scheme with the proximal point update can be written as

$$
\theta_{i_{p}}^{p}=\underset{\theta_{i_{p}}}{\arg \min }\left[E\left(\theta_{i_{p}}, \theta_{\neq i_{p}}^{p-1}\right)+\frac{1}{2 a_{i_{p}}^{p-1}}\left\|\theta_{i_{p}}-\theta_{i_{p}}^{p-1}\right\|_{2}^{2}\right]
$$

where $\frac{1}{2 a_{i_{p}}^{p-1}}\left\|\theta_{i_{p}}-\theta_{i_{p}}^{p-1}\right\|_{2}^{2}$ is the so-called quadratic proximal term and $a_{i_{p}}^{p-1}$ serves as a step size and can be any bounded positive number. The addition of the quadratic proximal term makes the function of each sub-problem dominate the original objective around the current iteration and therefore produces increased stability and better convergence properties, especially in the case of nonsmooth optimization [25].

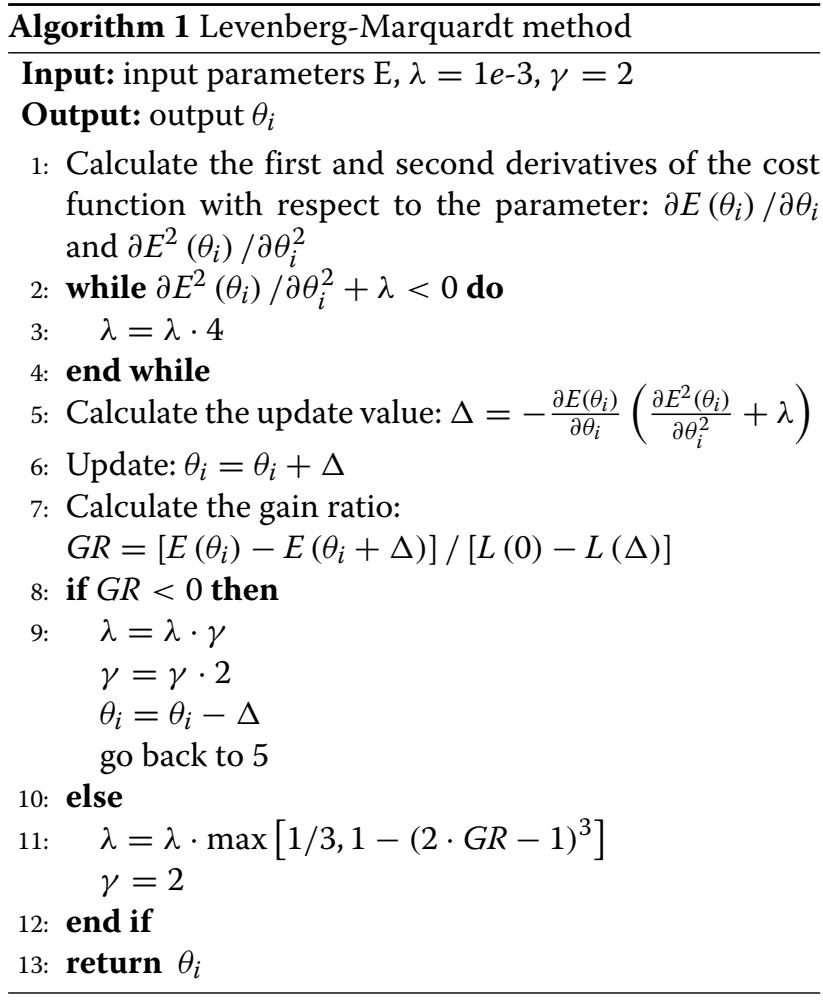

For the one-dimensional search in the CDA, the LM algorithm, which has been the de facto standard for most optimization problems [26], is utilized. In the LM method, the cost function in the neighborhood of the current iteration $\theta_{i}$ can be approximated as

$E\left(\theta_{i}+\Delta\right) \approx L(\Delta)=E\left(\theta_{i}\right)+\frac{\partial E\left(\theta_{i}\right)}{\partial \theta_{i}} \cdot \Delta+\frac{1}{2} \frac{\partial E^{2}\left(\theta_{i}\right)}{\partial \theta_{i}^{2}} \Delta^{2}$

where $\Delta$ is the update value and $L(\Delta)$ represents the approximation of $E\left(\theta_{i}+\Delta\right)$.

With two parameters, i.e., the damping parameter $\lambda$ and the division factor $\gamma$, the LM procedure can be summarized as in Algorithm 1, where the initial values of $\lambda$ and $\gamma$ are empirically obtained.

It can be seen from Algorithm 1 that the first and second derivatives of the cost functions are needed to complete the LM method, as illustrated in the Appendix.

Assume that the number of sub-apertures is $S N$; therefore, there are $M \cdot S N$ echoes in total. The envelope deviations obtained by parameter estimation can be written as

$\Delta_{\text {sub }}=\left[v_{1} \mathbf{t}_{\mathbf{s} 1}+a_{1} \mathbf{t}_{\mathbf{s} \mathbf{1}}{ }^{2}, \ldots, v_{S N} \mathbf{t}_{\mathbf{s S N}}+a_{S N} \mathbf{t}_{\mathbf{s S N}}{ }^{2}\right]^{T}$

where $v_{i}$ and $a_{i}(i=1,2, \ldots, S N)$ denote the estimated parameters and $\mathbf{t}_{\mathbf{s m}}(m=1,2, \ldots, M)$ represents the slow-time vector with length $M$ of each sub-aperture. Therefore, the total length of vector $\Delta_{\text {sub }}$ is $M \cdot S N$. 


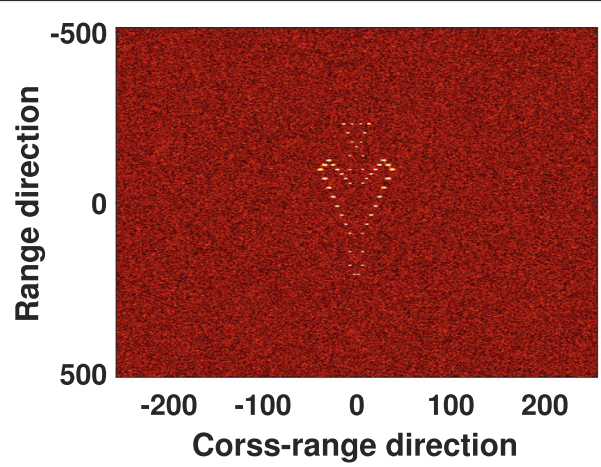

(a)

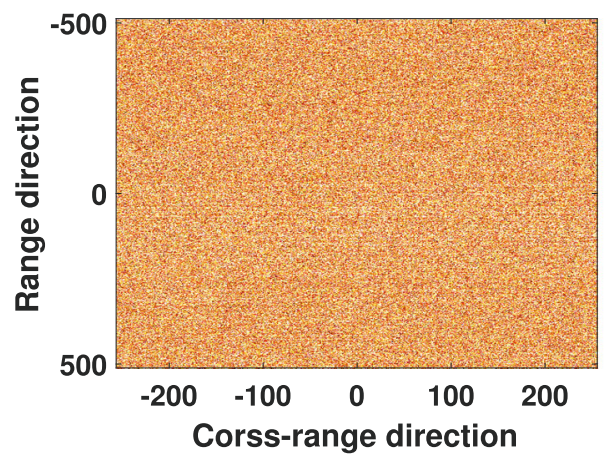

(c)

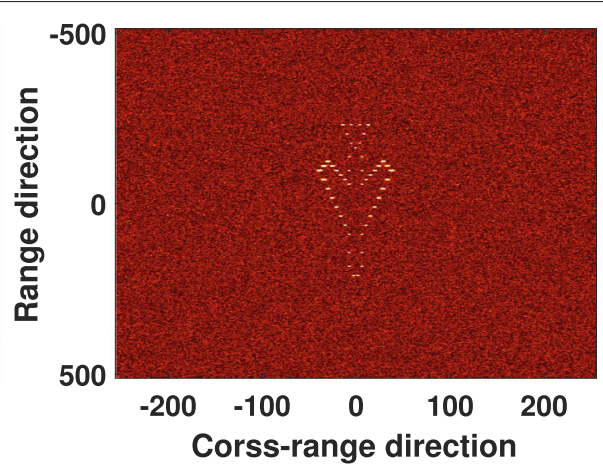

(b)

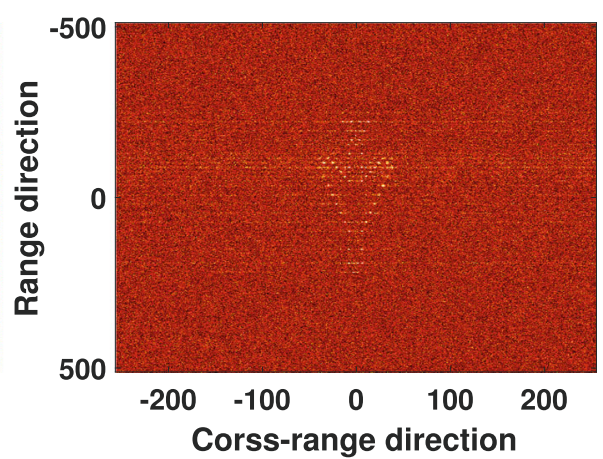

(d)

Fig. 7 Imaging results of the different methods under a - $15 \mathrm{~dB}$ SNR. a The ideal ISAR image, $\mathbf{b}$ the imaging result of the proposed algorithm, $\mathbf{c}$ the imaging result of the ACM, and $\mathbf{d}$ the imaging result of the MEARP

\subsection{Aligning the ARPs of the sub-apertures}

After the parameter estimation based on the method mentioned in the previous subsection and compensation according to the estimated results, the envelopes in each sub-aperture have been aligned. However, due to the following two reasons, some fine-tuned techniques are required to achieve better alignment.

On the one hand, the parameter estimation method focuses mainly on the alignment in the sub-apertures, and as a result, there exist envelope fluctuations among different sub-apertures; on the other hand, after the misalignment compensation in each sub-aperture, the processing gain of non-coherent integration can be obtained by means of averaging all envelopes in a sub-aperture, which provides useful information for performance enhancement in low SNR scenarios.

To make full use of the non-coherent integration gain and improve the effect of alignment between subapertures, we implement the ACM (5) on the ARPs of all sub-apertures. The framework of this fine-tuned technique is demonstrated below.

Suppose the estimated motion parameters of the $k$ th sub-aperture are $\hat{v}$ and $\hat{a}$; therefore, the compensated echoes of this sub-aperture can be written as

$$
x_{c k}\left(t_{m}, t_{n}\right)=\tilde{x}_{k}\left(t_{m}, t_{n}\right) \cdot \exp \left[j 2 \pi\left(\hat{v} t_{m}+\hat{a} t_{m}^{2}\right)\right]
$$

where $x_{c k}\left(t_{m}, t_{n}\right)$ denotes the aligned echoes of the $k$ th sub-aperture. The ARP of the $k$ th sub-aperture can be expressed as

$$
h=\frac{1}{M} \sum_{m=0}^{M-1}\left|f f t\left\{x_{c k}\left(t_{m}, t_{n}\right)\right\}\right|
$$

Again, $M$ is the number of echoes in one sub-aperture, and $f f t\{\cdot\}$ represents application of the FFT along the fast-time direction.

Assume that the number of sub-apertures is $S N$. After the ACM, the $S N$ values of the envelope deviations are obtained, i.e.,

$$
\Delta_{\text {ave }}=\left[\Delta_{1}, \Delta_{2}, \ldots, \Delta_{S N}\right]^{T}
$$

Table 2 Comparison of simulated imaging results under a -15 dB SNR

\begin{tabular}{lllll}
\hline & Original image & Proposed method & ACM & MEARP \\
\hline Entropy & 12.62062 & 12.62458 & 12.72116 & 12.68513 \\
Contrast & 0.56509 & 0.56332 & 0.54001 & 0.54963 \\
\hline
\end{tabular}


where $\Delta_{i}(i=1,2, \ldots, S N)$ represents the envelope deviation of each sub-aperture's ARP and $\Delta_{\text {ave }}$ denotes the vector of all deviations.

The length of vector $\Delta_{\text {ave }}$ should be extended to $M$ when carrying out compensation for each echo. The longer version of $\Delta_{\text {ave }}$ can be expressed as

$$
\Delta_{\text {ave }}=[\underbrace{\overbrace{\Delta_{1}, \ldots, \Delta_{1}}^{M}, \ldots, \underbrace{\Delta_{S N}, \ldots, \Delta_{S N}}_{M}}_{M}]^{T}
$$

\subsection{Total error fitting using locally weighted regression}

In the previous two subsections, the envelope deviations in each sub-aperture and between every two subapertures were obtained through optimization and the ACM, respectively. With the combination of (17) and (21), the total envelope deviations can be expressed as

$$
\Delta_{\text {total }}=\Delta_{\text {sub }}+\Delta_{\text {ave }}
$$

where $\Delta_{\text {total }}$ denotes the total deviations of the envelopes.

After aligning the ARPs of all sub-apertures, in general, the misaligned envelopes can be calibrated well. To achieve a higher performance, some fine tuning is still required. Figure 1 shows an estimation result for a full aperture's envelope misalignment error, where relatively accurate error estimation could be achieved; however, according to the enlarged error estimation curve, there exist step changes between the two sub-apertures, which can undermine the imaging quality. Because each subaperture is aligned as a whole by the method proposed in the previous subsection, the step changes are inevitable.

These step changes can be easily smoothed and eliminated by some curve-fitting techniques. By referring to [27] and [11], we use locally weighted regression (LOESS) to smooth the step changes between every two adjacent sub-apertures. The procedure of LOESS is briefly introduced in the following:

1 Suppose that there are $N$ points to be fitted, i.e., $\left[x_{1}, \ldots, x_{N}\right]^{T}$. For the $i$ th point $x_{i}$, put $N \cdot f_{r}\left(0<f_{r} \leq 1\right)$ points into its neighborhood $\Omega_{i}$.

2 Determine the weight $w_{k}\left(x_{i}\right), k=1,2, \ldots, N \cdot f_{r}$ for the weighted least squares (WLS) in the neighborhood of $x_{i}$ using tricube functions.

3 Because of the short length of the neighborhood, it is reasonable to model the points in the neighborhood as quadratic. After conducting WLS, the $i$ th fitted value can be expressed as $y_{i}=\beta_{0}+\beta_{1} x_{i}+\beta_{2} x_{i}^{2}$, where $\beta_{i}, i=0,1,2$ are the estimated coefficients of the quadratic polynomial.

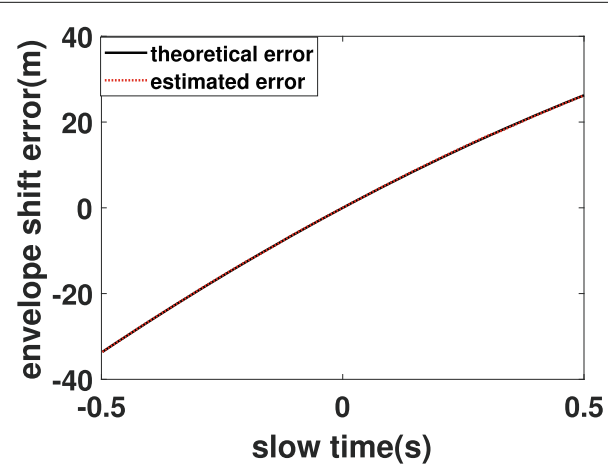

(a)

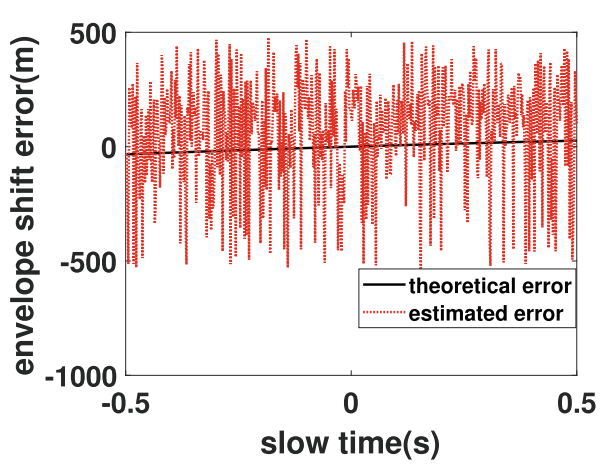

(b)

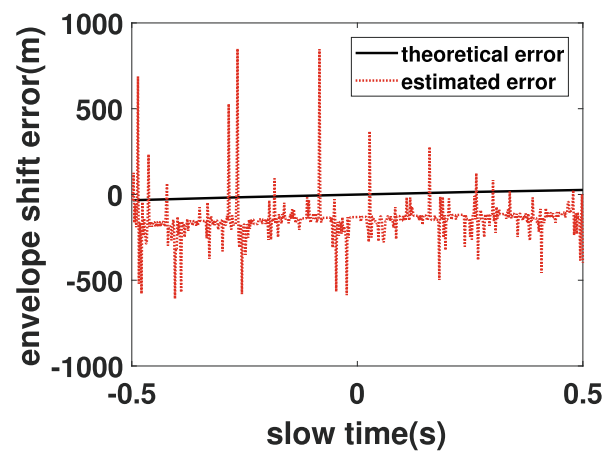

(c)

Fig. 8 Estimated error curves of the three methods under a - 15 dB SNR. a The estimated error curve of the proposed algorithm, $\mathbf{b}$ the estimated error curve of the ACM, and $\mathbf{c}$ the estimated error curve of the MEARP 
4. Repeat steps $1-3$ for all $N$ points to obtain $N$ fitted values.

After LOESS, accurate envelope deviations can be obtained, and good alignment can be achieved. The whole framework is shown in Fig. 2.

\subsection{Optimal selection and computational complexity}

This subsection discusses how to choose the number of sub-apertures and the computational complexity of the proposed algorithm.

By referring to [28] and [29], we develop the following adaptive selection method:

1 Initialize $S N$. The principle of initializing $S N$ is that the envelope deviation in one sub-aperture should not exceed half the range unit, i.e., $c / 4 F_{s}$, where $c$ is the velocity of light and $F_{S}$ is the sampling rate.

2 Implement the minimum entropy optimization. The estimated error of the $p$ th sub-aperture is $\Delta R_{p}\left(t_{m}\right)$, where $t_{m}$ represents the slow time of the current aperture.

3 Double $S N$ and implement the minimum entropy optimization. The pth aperture in (2) is split into two equal sub-apertures, and the estimated envelope errors in each one can be expressed as $\Delta R_{p 1}\left(t_{m}\right)$ and $\Delta R_{p 2}\left(t_{m}\right)$. They can be denoted as

$\Delta R_{p N e w}\left(t_{m}\right)=\left[\Delta R_{p 1}\left(t_{m}\right), \Delta R_{p 2}\left(t_{m}\right)\right]$, and the length of $t_{m}$ is equal to that in (2).

4. If the following condition is satisfied, the initialized $S N$ can be used; otherwise, return to (3) and repeat.

$$
\begin{aligned}
& \mid \max \left(\Delta R_{p}\left(t_{m}\right)-\Delta R_{p N e w}\left(t_{m}\right)\right) \\
& -\min \left(\Delta R_{p}\left(t_{m}\right)-\Delta R_{p N e w}\left(t_{m}\right)\right) \mid \leq c / 4 F_{s}
\end{aligned}
$$

In addition to the selection scheme above, in real cases, it is also important to jointly consider prior information, such as the motion parameters, the expected gain through accumulated echoes, and the computational burden (a larger number of apertures means a higher computational complexity). Moreover, when the accumulated echoes are insufficient, a certain number of echoes can be reused by two different sub-apertures. In general, the selection of the number of sub-apertures requires thorough consideration.

In the following part of this subsection, the computational complexity is briefly numerically analyzed, with the detailed derivation shown in the Appendix. As mentioned above, the proposed algorithm contains three parts. In the first part, we use the CDA to solve the optimization in

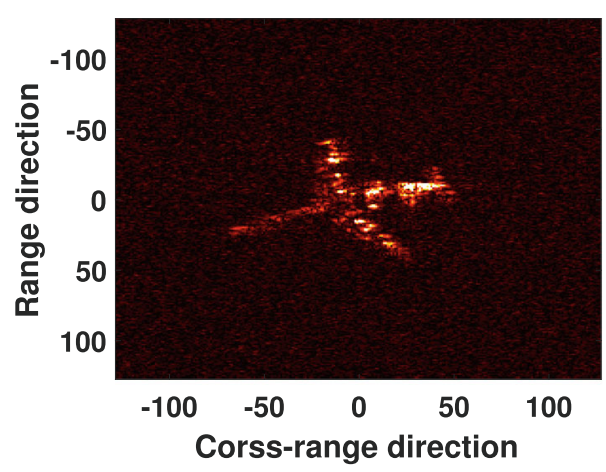

(a)

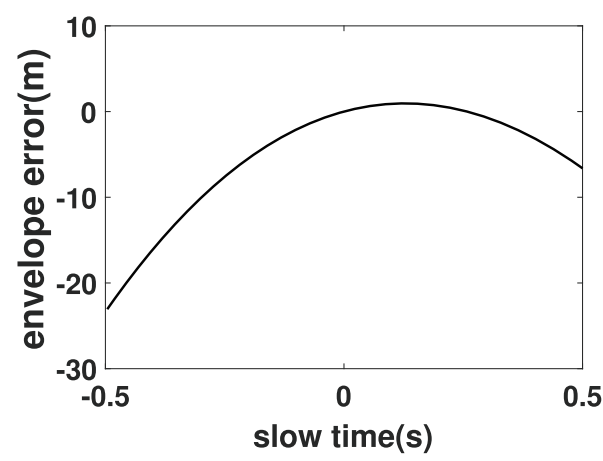

(c)

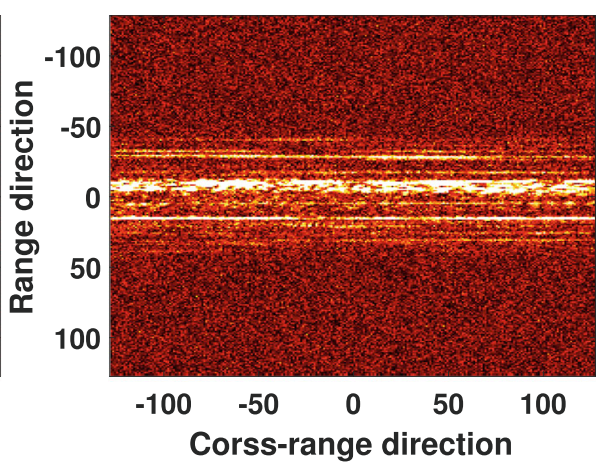

(b)

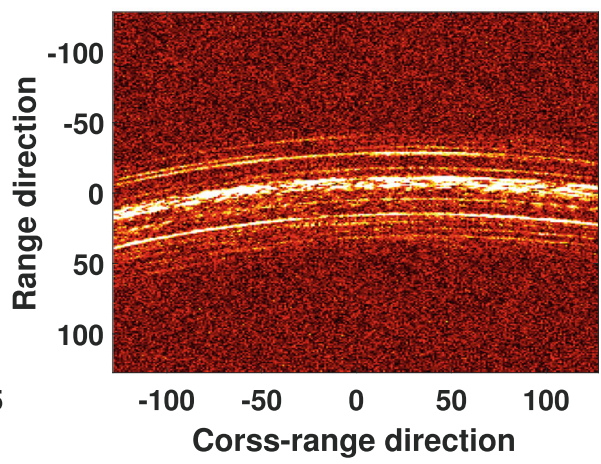

(d)

Fig. 9 Adding the envelope deviation errors. a The original ISAR image, b the original range profiles, $\mathbf{c}$ the three-order error, and $\mathbf{d}$ the contaminated range profiles 
each sub-aperture. In each loop, the computational burden is devoted mainly to obtaining the entropy and its first and second derivatives. The computational complexity of these operations is denoted as

$$
\begin{aligned}
& N_{\text {mul }}^{O P} \sim \Theta\left(M \cdot N \cdot \log _{2} N\right) \\
& N_{\text {add }}^{O P} \sim \Theta\left(M \cdot N \cdot \log _{2} N\right)
\end{aligned}
$$

where $N_{m u l}^{O P}$ and $N_{a d d}^{O P}$ represent the numbers of multiplications and additions of the proposed algorithm's optimization procedure, respectively, and $M$ and $N$ denote the numbers of Doppler and range cells of each sub-aperture, respectively. It also can be seen that each sub-aperture is independent; thus, parallel programming can be used to execute all optimizations concurrently.

The second step is the ACM in each loop, of which the computational burden arises mainly from obtaining the correlation function between the current profile and the reference. The computational complexity of this step is

$$
\begin{aligned}
& N_{\text {mul }}^{A C M} \sim \Theta\left(S N \cdot N \cdot \log _{2} N\right) \\
& N_{\text {add }}^{A C M} \sim \Theta\left(S N \cdot N \cdot \log _{2} N\right)
\end{aligned}
$$

where $N_{m u l}^{A C M}$ and $N_{\text {add }}^{A C M}$ represent the numbers of multiplications and additions of the proposed algorithm's ACM procedure, respectively, $N$ denotes the number of range cells of each sub-aperture, and $S N$ is the number of subapertures.

With reference to [30], LOESS also decomposes the problem into independent pieces, with all operations being completely parallel. Therefore, with well-designed concurrent programming, the computational complexity of the LOESS step is equal to that of a weighted least squares operation with few points.

\section{Results and discussion}

In this section, experiments based on simulated and real measured data are reported to verify the effectiveness of the anti-low-SNR characteristics of the proposed algorithm. Entropy and contrast were chosen to evaluate the quality of the range profiles and ISAR images. The ACM (5) and MEARP (6) were also used for comparison. The computation platform was based on the Windows 10 64bit operating system, an Intel Core i5-9300H@2.40 GHz CPU, 8 GB of memory, and MATLAB version 2017b.

\subsection{Simulated experiment}

In this subsection, a simple plane model, as shown in Fig. 3a, is used as the target of the simulated experiments. The radar system has a $15-\mathrm{GHz}$ carrier frequency and $2-\mathrm{GHz}$ bandwidth. The numbers of fast-time sampling points and accumulated echoes are both 512 . Figure $3 \mathrm{~b}$ is the original ISAR image of this plane model. Figure $3 \mathrm{c}$ and d demonstrate the original (without misalignment) and the misaligned HRRPs.

To preliminarily verify the effectiveness of the proposed algorithm using this simulated data, we added extra white Gaussian noise to change the SNR to -10 and $-13 \mathrm{~dB}$.

First, the alignment results, the final ISAR images and their evaluations, and the estimated error curve under $-10 \mathrm{~dB}$ SNR are given. For the purpose of comparison, these results obtained by the proposed algorithm, ACM, and MEARP are all displayed.

Figure 4 gives the aligned HRRPs under the $-10 \mathrm{~dB}$ SNR obtained through the three methods above. It can be seen that due to the strong background noise, the maximum-correlation-based algorithm has the worst alignment result and that a few profiles at the beginning of the slow time cannot be identified. The proposed and MEARP algorithms utilize the global information so that

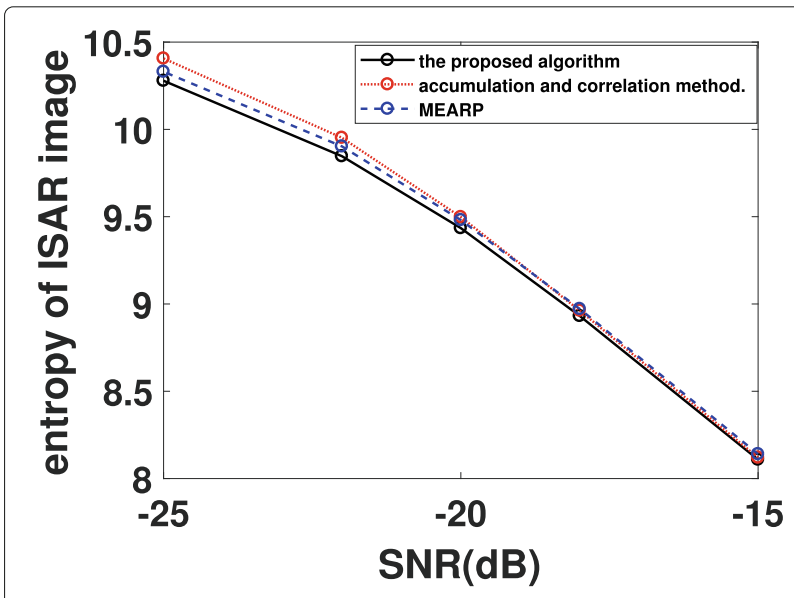

(a)

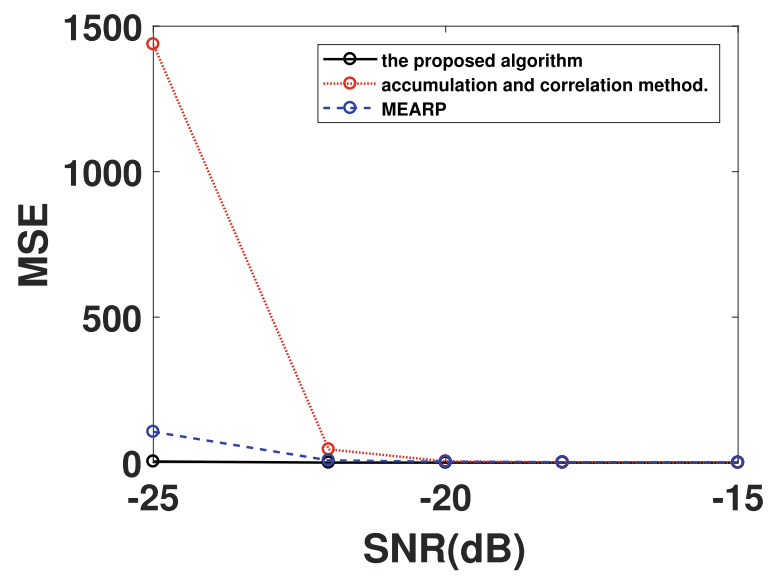

(b)

Fig. 10 Results of the Monte Carlo experiments. a The mean entropy of ISAR images obtained from the three methods and $\mathbf{b}$ the MSE of the error estimation 
their range profiles have higher sharpness than those of the ACM.

The theoretical and estimated envelope deviation curves are given in Fig. 5 to compare the accuracies of the three methods. In Fig. 5, the dotted lines are the estimated curves, and the full lines are the theoretical ones. It can be seen from the first sub-figure that the estimated curve is almost consistent with the theoretical one, indicating the high accuracy of the proposed algorithm under low SNR situations. However, there exist several abrupt changes in the estimation of the ACM, which is the result of the noise interference. The results of the MEARP, with fewer abrupt changes than those of the ACM, reveal an overall shift of the envelopes and are still not as good as those of the proposed algorithm.

As shown in Fig. 4, it is difficult to distinguish the pictures of the range profiles under strong noise. Moreover, under very low SNRs, the entropy of the range profiles cannot fully reflect the degree of focus due to the noise disturbance. Therefore, the final image is obtained, and the image quality is used as another reference for evaluation. After the range alignment, by using the autofocusing method proposed in [31], we can obtain the final ISAR image. Figure 6 demonstrates the ISAR imaging results of the three methods. The qualities of the images evaluated by the entropy and contrast are shown in Table 1 . Through Fig. 6 and Table 1, it can be seen that the proposed algorithm can obtain the best degree of focus, namely, the lowest entropy and highest contrast. It should also be noted that the ACM performs worse than the two other algorithms, with some scatter points overwhelmed by the ground noise.

In more challenging situations ( $-15 \mathrm{~dB} \mathrm{SNR})$, the proposed algorithm can still achieve high performance. Figure 7 shows an ISAR image under $-15 \mathrm{~dB}$ for the three methods. Their evaluations, conducted using the entropy and contrast, are listed in Table 2. As is the case in the $-10 \mathrm{~dB}$ SNR situation, the proposed algorithm achieves the highest performance. It can be seen in Fig. 7 that the ACM cannot image at all and that some scatters drowned in noise in the result of the MEARP can hardly be identified. However, the imaging result of the proposed algorithm is almost the same as the original one. Through Table 2, it can be seen that compared with the qualities of the results of the ACM and MEARP, the proposed algorithm achieves the highest contrast and lowest entropy, indicating that the proposed algorithm can produce the most-focused images.

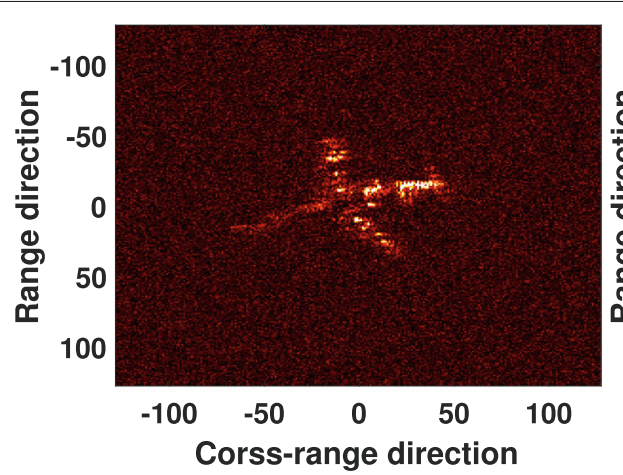

(a)

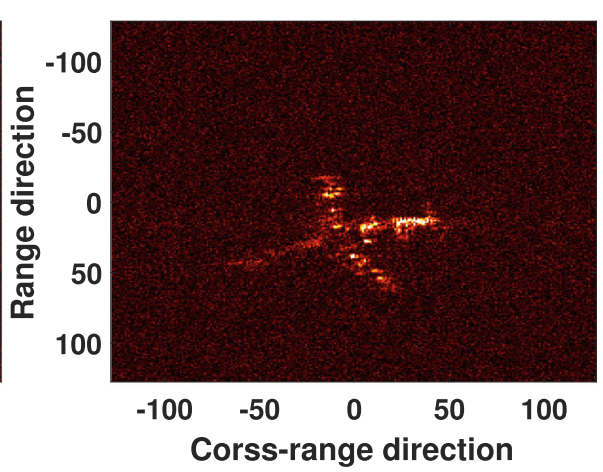

(b)

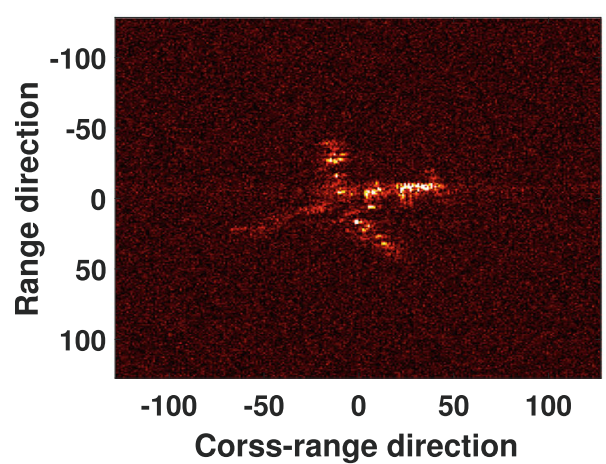

(c)

Fig. 11 Imaging results under a - $20 \mathrm{~dB}$ SNR. a The imaging result of the proposed algorithm, $\mathbf{b}$ the imaging result of the ACM, and $\mathbf{c}$ the imaging result of the MEARP 
The estimated error curves obtained through the three methods under $-15 \mathrm{~dB}$ are also demonstrated. As in Fig. 5, in Fig. 8, the proposed algorithm realizes the highest accuracy.

\subsection{Experiment on real measured data}

Real measured Yak-42 data were adopted to validate the performance of the proposed algorithm. The radar recording the dataset was a $\mathrm{C}$-band ISAR experimental system with a $400-\mathrm{MHz}$ bandwidth, 5.52-GHz carrier frequency, $100-\mathrm{Hz}$ PRF, and 25.6- $\mu$ s pulse width. The numbers of fast-time sampling points and accumulated echoes were both 256. The SNR of the original echoes (before range compression) was $-15 \mathrm{~dB}$. To test the robustness of the proposed algorithm in low SNR scenarios, extra white Gaussian noise was added, making the SNR of echoes $-20,-22$, and $-25 \mathrm{~dB}$. Monte Carlo experiments were performed under these low SNRs.

The three-order envelope deviation error, which can be expressed as (27), was added:

$$
R_{\text {error }}=15 t_{s}-60 t_{s}^{2}+7 t_{s}^{3}
$$

where $R_{\text {error }}$ is the added error and $t_{s}$ represents the slow time.
The original ISAR image, the range profiles of 256 echoes, the added error, and the contaminated range profiles are demonstrated in Fig. 9. In the following part of this subsection, the performances of the proposed algorithm under $-20,-22$, and $-25 \mathrm{~dB}$ SNR are evaluated. The imaging results and estimated error curves of the three algorithms are demonstrated. To verify the robustness of the proposed algorithm in low SNR scenarios, Monte Carlo experiments were conducted.

Figure 10 demonstrates the ISAR image entropy and MSE of the error estimation achieved after the 150 Monte Carlo experiments. In Fig. 10, the full lines, dotted lines, and dashed lines indicate the results of the proposed algorithm, ACM, and MEARP, respectively. It can be seen in Fig. 10a that the entropy of the ISAR images obtained from the three methods indicates that the proposed algorithm achieved lower entropy than did the ACM and MEARP under -15 to approximately $-25 \mathrm{~dB}$ SNRs. Furthermore, according to Fig. 10b, the proposed algorithm had the lowest MSE of error estimation, which further verifies its robustness in low SNR scenarios. Through Fig. 10a and b, it can be seen that the MEARP performed better than the ACM under -20 to approximately $-25 \mathrm{~dB}$ SNRs, which is consistent with the conclusion in [7].

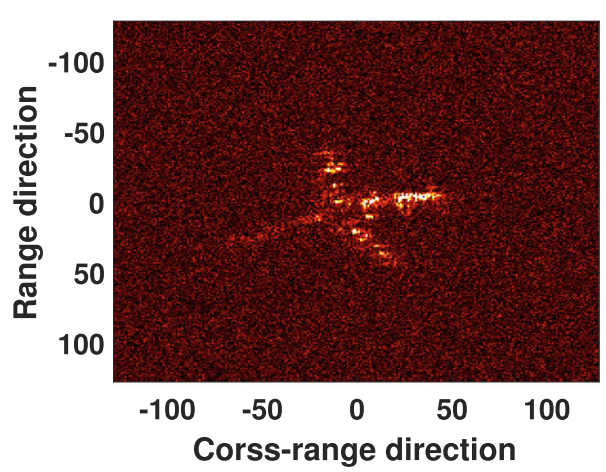

(a)

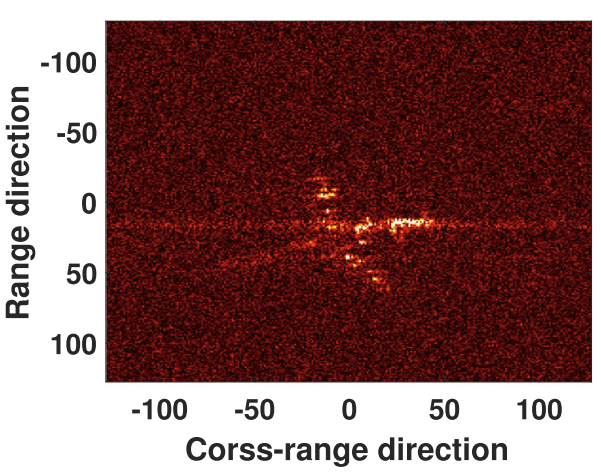

(b)

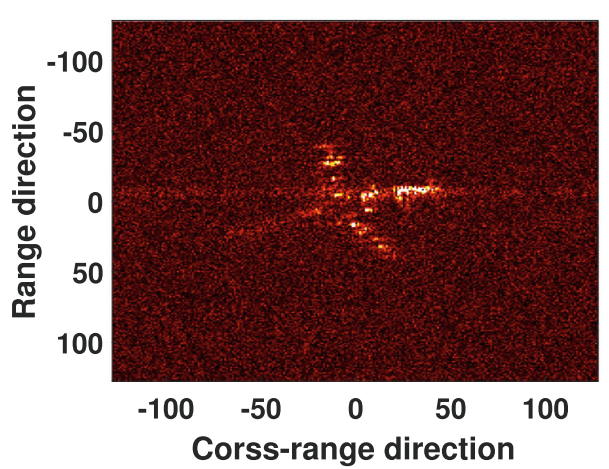

(c)

Fig. 12 Imaging results under a $-22 \mathrm{~dB}$ SNR. a The imaging result of the proposed algorithm, $\mathbf{b}$ the imaging result of the ACM, and $\mathbf{c}$ the imaging result of the MEARP 
Figures 11, 12, and 13 demonstrate the ISAR imaging results of the three methods under $-20,-22$, and $-25 \mathrm{~dB}$ SNRs, respectively. The first sub-figures in these figures show the results of the proposed algorithm, and the second and third sub-figures show the results of the ACM and MEARP, respectively. It can be seen from Figs. 11, 12, and 13 that for extremely low SNRs, especially -22 and $-25 \mathrm{~dB}$, severe defocus occurred in the images yielded by the ACM and MEARP, and the outlines of the planes could not be distinguished. However, the imaging results of the proposed algorithm remained clear and well focused as the background noise increased. Therefore, through Figs. 11, 12, and 13, it can be concluded that in low SNR scenarios, the proposed algorithm can realize the most-focused ISAR images compared with the ACM and MEARP.

In Figs. 14, 15, and 16, the error estimation curves of the three algorithms under $-20,-22$, and $-25 \mathrm{~dB}$ SNRs are demonstrated. The full lines represent the theoretical results, and the dotted lines represent the estimations. It can be seen that compared with the ACM and MEARP, the proposed algorithm is the most robust and has the highest estimation accuracy. The estimated error curve obtained by the proposed algorithm is approximately consistent with the theoretical one; however, there exists a very large estimation error in the curves generated by the ACM and MEARP, which will cause severely defocused images. Therefore, through Figs. 14, 15, and 16, the superiority of the proposed algorithm in low SNR scenarios is further confirmed.

\section{Conclusions}

In low SNR scenarios, it is challenging to realize range alignment in ISAR translation motion compensation. Illaligned range profiles can cause imprecise phase compensation results. A novel range alignment method was proposed in this article. First, the target motion information was adopted as useful information, and the full aperture was split into several sub-apertures. In each subaperture, the minimum entropy principle was applied as a metric, and the CDA with a proximal point updating scheme was implemented as the solver to estimate the motion parameters (i.e., velocity and acceleration) in each sub-aperture. Second, after parameter estimation in every sub-aperture, the non-coherent accumulation gain was obtained by averaging the profiles in each sub-aperture,

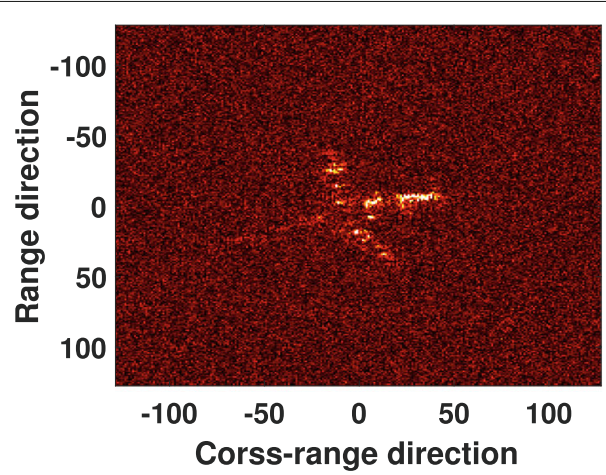

(a)

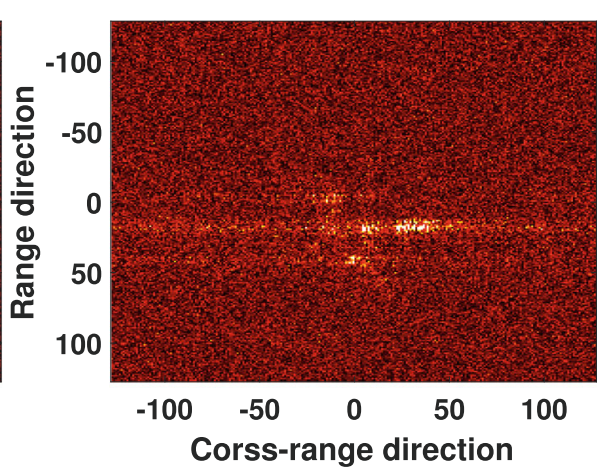

(b)

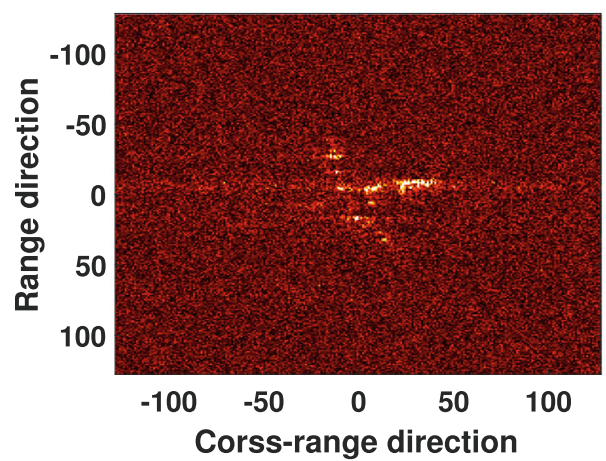

(c)

Fig. 13 Imaging results under a $-25 \mathrm{~dB}$ SNR. a The imaging result of the proposed algorithm, $\mathbf{b}$ the imaging result of the ACM, and $\mathbf{c}$ the imaging result of the MEARP 


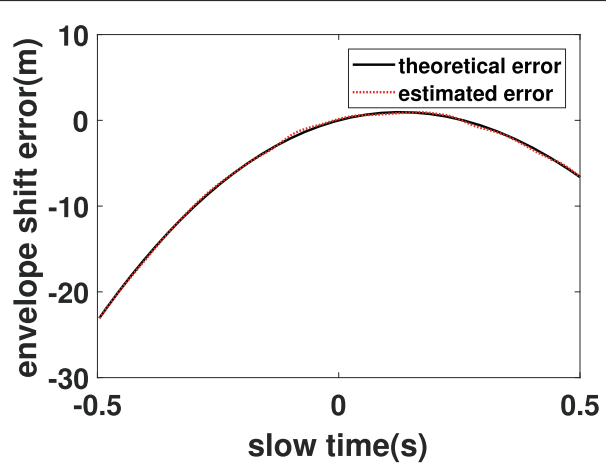

(a)

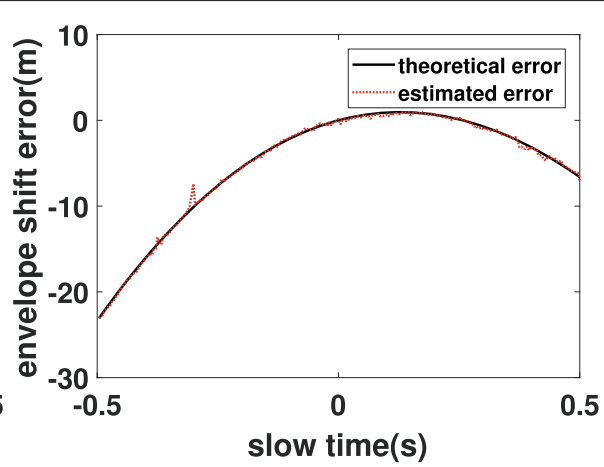

(b)

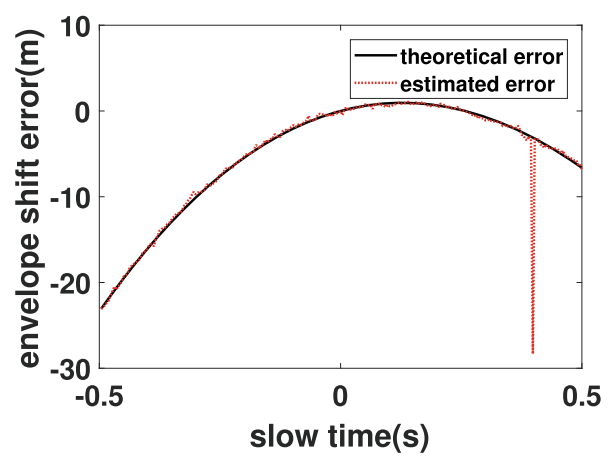

(c)

Fig. 14 Estimation results under a $-20 \mathrm{~dB}$ SNR. a The estimation result of the proposed algorithm, $\mathbf{b}$ the estimation result of the $A C M$, and $\mathbf{c}$ the estimation result of the MEARP

which could be used as further information to improve the anti-noise characteristics. The first step focused mainly on alignment in each sub-aperture; therefore, we used the ACM to align the ARPs of each sub-aperture. Third, to eliminate the step change in the total estimated error curve, LOESS was applied to smooth the discontinuity. Experiments based on real measured Yak-42 data were conducted, and the classic ACM and MEARP were used for comparison. The results show that the proposed algorithm achieved the best performance in low SNR scenarios. Further work will be focused on parallelization of the proposed algorithm to use it in real-time ISAR scenarios.

\section{Appendix}

Proof of the first and second derivatives of the cost function In this section, the first and second derivatives of (12) are given.

The ARP is a one-dimensional function of the unknown parameters $v$ and $a$. A vector containing an unknown is denoted as $\boldsymbol{\theta}=[v, a]^{T}$. Combined with (11) and (13), the first derivative of (12) can be written as

$$
\frac{\partial}{\partial \theta_{i}} E(A R P)=-\frac{1}{S_{A R P}} \sum_{n=0}^{N-1}\left(1+\ln |A R P|^{2}\right) \frac{\partial|A R P|^{2}}{\partial \theta_{i}}
$$

where $\theta_{i}(i=1,2)$ is the $i$ th element of $\boldsymbol{\theta}$.

Therefore,

$\frac{\partial A R P^{2}}{\partial \theta_{i}}=2 A R P \cdot \frac{\partial A R P}{\partial \theta_{i}}=2 A R P \cdot \frac{1}{M} \sum_{m=0}^{M-1} \frac{\partial\left|f_{k}(\boldsymbol{\theta})\right|}{\partial \theta_{i}}$

It can be seen from (28) and (29) that $\partial\left|f_{k}(\boldsymbol{\theta})\right| / \partial \theta_{i}$ should first be deduced to obtain $\partial E(A R P) / \partial \theta_{i}$.

Consider that

$$
\begin{aligned}
& \frac{\partial f_{k}}{\partial v}=f f t\left\{\tilde{x}_{k}\left(t_{m}, \hat{t}\right) \cdot\left(-j 2 \pi t_{m} \hat{t}\right)\right\} \\
& \frac{\partial f_{k}}{\partial a}=f f t\left\{\tilde{x}_{k}\left(t_{m}, \hat{t}\right) \cdot\left(-j 2 \pi t_{m}^{2} \hat{t}\right)\right\}
\end{aligned}
$$




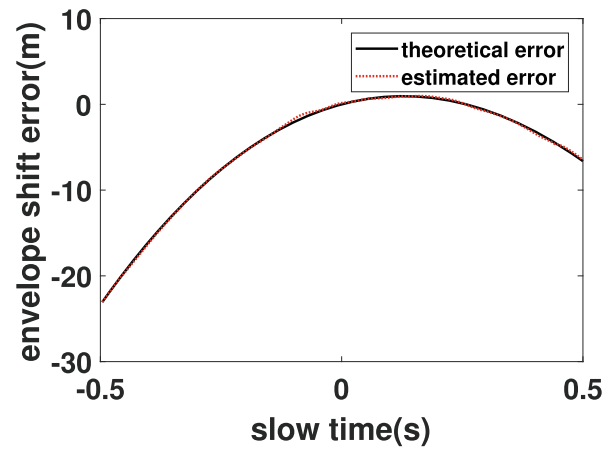

(a)

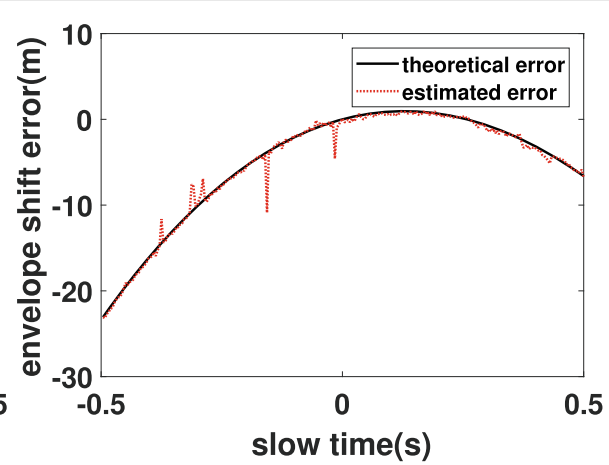

(b)

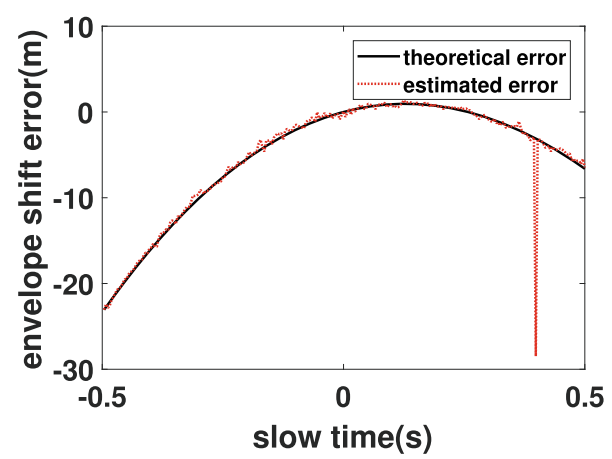

(c)

Fig. 15 Estimation results under a $-22 \mathrm{~dB}$ SNR. a The estimation result of the proposed algorithm, $\mathbf{b}$ the estimation result of the ACM, and $\mathbf{c}$ the estimation result of the MEARP

and

$$
\begin{aligned}
\frac{\partial\left|f_{k}(\boldsymbol{\theta})\right|}{\partial \theta_{i}} & =\frac{\partial\left[f_{k} \cdot f_{k}{ }^{*}\right]^{1 / 2}}{\partial \theta_{i}} \\
& =\frac{1}{2}\left[f_{k} \cdot f_{k}^{*}\right]^{-1 / 2} \cdot \frac{\partial f_{k} \cdot f_{k}{ }^{*}}{\partial \theta_{i}} \\
& =\frac{1}{2}\left[f_{k} \cdot f_{k}^{*}\right]^{-1 / 2} \cdot\left[f_{k} \frac{\partial f_{k}^{*}}{\partial \theta_{i}}+f_{k}^{*} \frac{\partial f_{k}}{\partial \theta_{i}}\right] \\
& =\left[f_{k} \cdot f_{k}^{*}\right]^{-1 / 2} \cdot \operatorname{Re}\left\{f_{k}^{*} \frac{\partial f_{k}}{\partial \theta_{i}}\right\} \\
& =\frac{1}{\left|f_{k}\right|} \cdot \operatorname{Re}\left\{f_{k}^{*} \frac{\partial f_{k}}{\partial \theta_{i}}\right\}
\end{aligned}
$$

where $f_{k}^{*}$ represents the conjugate of $f_{k}$.

Taking (29) (32) in (28), we can obtain an expression for the first derivative of (12).

The second derivative of (12) can be written as

$$
\begin{aligned}
\frac{\partial^{2} E(A R P)}{\partial \theta_{i}^{2}}= & -\frac{1}{S_{A R P}} \sum_{n=0}^{N-1}\left(1+\ln |A R P|^{2}\right) \frac{\partial^{2}|A R P|^{2}}{\partial \theta_{i}^{2}} \\
& -\frac{1}{S_{A R P}} \sum_{n=0}^{N-1} \frac{1}{|A R P|^{2}}\left(\frac{\partial|A R P|^{2}}{\partial \theta_{i}}\right)^{2}
\end{aligned}
$$

and

$$
\begin{aligned}
\frac{\partial^{2} A R P^{2}}{\partial \theta_{i}^{2}} & =2 \cdot\left(\frac{\partial A R P}{\partial \theta_{i}}\right)^{2}+2 A R P \cdot \frac{\partial^{2} A R P}{\partial \theta_{i}^{2}} \\
& =2 \cdot\left(\frac{1}{M} \sum_{m=0}^{M-1} \frac{\partial\left|f_{k}\right|}{\partial \theta_{i}}\right)^{2}+2 A R P \cdot \frac{1}{M} \sum_{m=0}^{M-1} \frac{\partial^{2}\left|f_{k}\right|}{\partial \theta_{i}^{2}}
\end{aligned}
$$

It can be seen from (33) and (34) that $\partial^{2}\left|f_{k}\right| / \partial \theta_{i}^{2}$ should first be deduced to obtain $\partial^{2} E(A R P) / \partial \theta_{i}^{2}$.

Consider that

$$
\begin{aligned}
& \frac{\partial^{2} f_{k}}{\partial v^{2}}=f f t\left\{\tilde{x}_{k}\left(t_{m}, t_{n}\right) \cdot\left(-4 \pi^{2} t_{m}^{2} t_{n}^{2}\right)\right\} \\
& \frac{\partial^{2} f_{k}}{\partial a^{2}}=f f t\left\{\tilde{x}_{k}\left(t_{m}, t_{n}\right) \cdot\left(-4 \pi^{2} t_{m}^{4} t_{n}^{2}\right)\right\}
\end{aligned}
$$

and

$$
\begin{aligned}
\frac{\partial^{2}\left|f_{k}(\theta)\right|}{\partial \theta_{i}^{2}}= & \frac{\partial}{\partial \theta_{i}}\left\{\frac{1}{2\left|f_{k}\right|}\left[f_{k} \frac{\partial f_{k}^{*}}{\partial \theta_{i}}+f_{k}^{*} \frac{\partial f_{k}}{\partial \theta_{i}}\right]\right\} \\
= & -\frac{1}{\left|f_{k}\right|^{2}} \frac{\partial\left|f_{k}\right|}{\partial \theta_{i}} \operatorname{Re}\left\{f_{k} * \frac{\partial f_{k}}{\partial \theta_{i}}\right\} \\
& +\frac{1}{\left|f_{k}\right|} \operatorname{Re}\left\{f_{k} * \frac{\partial^{2} f_{k}}{\partial \theta_{i}^{2}}+\left|\frac{\partial f_{k}}{\partial \theta_{i}}\right|^{2}\right\}
\end{aligned}
$$




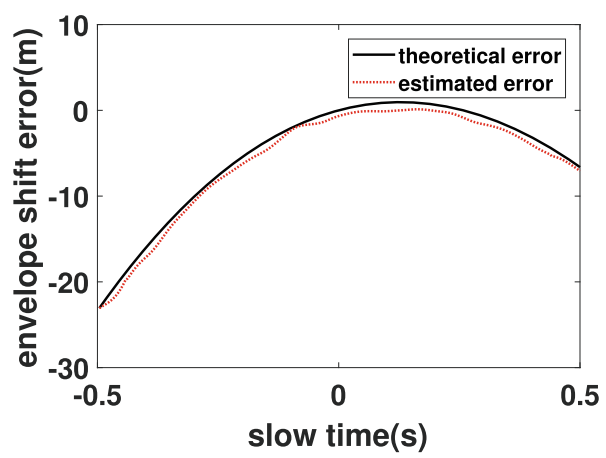

(a)

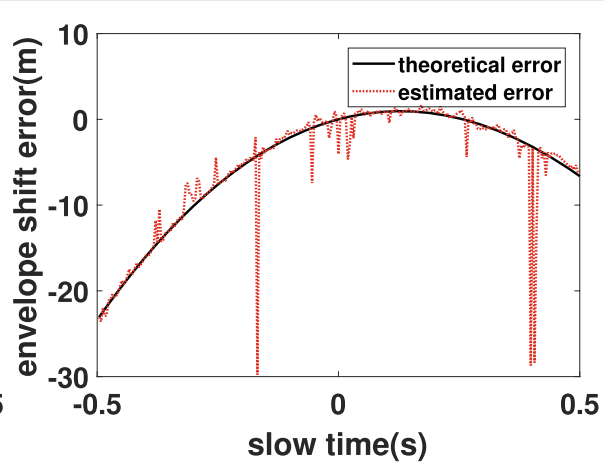

(b)

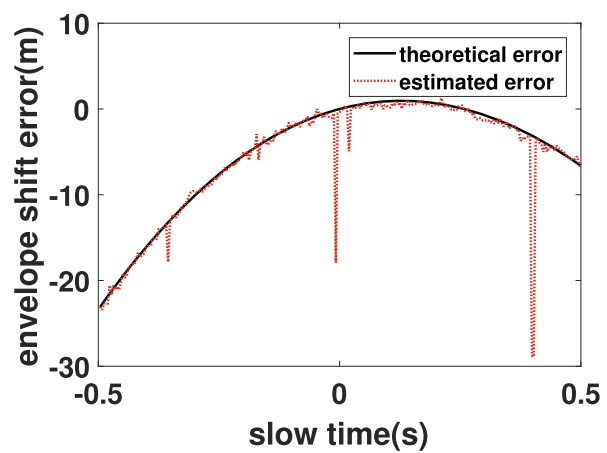

(c)

Fig. 16 Estimation results under a $-25 \mathrm{~dB}$ SNR. a The estimation result of the proposed algorithm, $\mathbf{b}$ the estimation result of the ACM, and $\mathbf{c}$ the estimation result of the MEARP

Taking (34) (37) in (33), we can obtain an expression for the second derivative of (12).

\section{Numerical computational complexity analysis}

As (11) shows, $f_{k}$ contains $M$ FFTs in the fast-time direction. Therefore, the numbers of multiplications and additions can be approximately expressed as

$$
\begin{aligned}
& N_{\text {mul }}^{(10)}=M\left(\frac{N}{2} \log _{2} N+N\right) \\
& N_{\text {add }}^{(10)}=M\left(N \log _{2} N+N\right)
\end{aligned}
$$

According to (30) and (31), the first derivative of $f_{k}$ also contains $M$ FFT operations, whose computational complexity can be written as

$$
\begin{aligned}
& N_{m u l}^{(1 s t)}=M\left(\frac{N}{2} \log _{2} N+N\right) \\
& N_{\text {add }}^{(1 s t)}=M \cdot N \log _{2} N
\end{aligned}
$$

Because the $A R P, S_{A R P}$, and $\partial A R P^{2} / \partial \theta_{i}$ are all functions of $f_{k}$ or $\partial f_{k} / \partial \theta_{i}$, once $f_{k}$ and $\partial f_{k} / \partial \theta_{i}$ have been obtained, (28) can be obtained. In other words, the computational complexity of (28) is the sum of the complexity of comput$\operatorname{ing} f_{k}, \partial f_{k} / \partial \theta_{i}$, and other multiplications and additions in
(28). The final result is

$$
\begin{aligned}
& N_{\text {mul }}^{(27)}=M\left(\frac{N}{2} \log _{2} N+N\right)+3 N+2 M \cdot N \\
& N_{\text {add }}^{(27)}=2 M \cdot N \log _{2} N+M \cdot N+3 M+N
\end{aligned}
$$

Once (28) is obtained, in (33), only $\partial^{2} A R P^{2} / \partial \theta_{i}^{2}$ must be recomputed. According to (34) (37), the numbers of multiplications and additions of (34) can be written as

$$
\begin{aligned}
& N_{\text {mul }}^{(33)}=M\left(\frac{N}{2} \log _{2} N+N\right)+N \\
& N_{\text {add }}^{(33)}=M \cdot N \log _{2} N+2 M \cdot N+N
\end{aligned}
$$

The total numbers of multiplications and additions of (33) are equal to the sum of the multiplications and additions of (34) and other necessary ones contained in (33). Therefore, the computational complexity of (33) can be expressed as

$$
\begin{aligned}
& N_{\text {mul }}^{(32)}=M\left(\frac{N}{2} \log _{2} N+N\right)+5 N \\
& N_{\text {add }}^{(32)}=M \cdot N \log _{2} N+3 M \cdot N+2 N
\end{aligned}
$$

Combining (42), (43), (46), and (47), it can be seen that (23) and (24) have been proved. 
For the computational burden of the ACM, the focus is on the correlation function in each loop. To obtain the correlation between the current profile and the template through an FFT, three N-point FFT/IFFT operations and one N-point conjugate multiplication must be performed, and the average of the aligned profiles must be taken. An N-point FFT contains $N \log _{2} \mathrm{~N} / 2$ multiplications and $\mathrm{N} \log _{2} \mathrm{~N}$ additions, and $\mathrm{N}$-point conjugate multiplication has $\mathrm{N}$ multiplications. Therefore, the total computational burden of the ACM can be approximately written as

$$
\begin{aligned}
& N_{\text {mul }}^{(A C M)}=S N\left(3 \frac{N}{2} \log _{2} N+2 N\right) \\
& N_{\text {add }}^{(A C M)}=S N\left(3 N \log _{2} N+N\right)
\end{aligned}
$$

Therefore, (25) and (26) are proved.

\section{Supplementary Information}

The online version contains supplementary material available at https://doi.org/10.1186/s13634-020-00709-z.

Additional file 1: The file containing the data of the Yak-42 plane can be opened by MATLAB.

\section{Abbreviations}

ISAR: Inverse synthetic aperture radar; SNR: Signal-to-noise ratio; HSACM: High-order symmetric accumulated cross-correlation method; CPI: Coherent processing interval; CDA: Coordinate descent algorithm; LM: Levenberg-Marquardt; MEARP: Minimization of the entropy of the average range profile; MCA: Maximum-correlation-based algorithm; LFM: Linear frequency modulation; RVP: Residual video phase; FFT: Fast Fourier transform; HRRP: High-resolution range profile; ACM: Accumulation and correlation method; ARP: Average range profile; LOESS: Locally weighted regression

\section{Acknowledgements}

The authors thank the anonymous reviewers for their enlightening comments and careful review.

\section{Authors' contributions}

$Y L$ was a major contributor in writing the manuscript and carried out the simulation work. JY designed the major workflow of the proposed algorithm. $Y Z$ helped to improve the source code and modified some details of this algorithm. SX and the authors read and approved the final manuscript .

\section{Funding}

This work was supported in part by the National Natural Science Foundation of China under Grant 61571449 and Grant 61501471.

\section{Availability of data and materials}

The data of the Yak-42 plane is presented in the additional supporting files.

\section{Competing interests}

The authors declare that they have no competing interests.

\section{Author details \\ ${ }^{1}$ National Key Laboratory of Science and Technology on Automatic Target Recognition, National University of Defense Technology, Deya Road, Changsha 410073, China. ${ }^{2}$ Rocket Force University of Engineering, Xi'an 710025, China. ${ }^{3}$ School of Electronics and Communication Engineering, Sun Yat-sen University, Xingangxi Road, Guangzhou 510275, China.}

Received: 26 June 2020 Accepted: 30 November 2020

Published online: 11 January 2021

\section{References}

1. C.-C. Chen, H. C. Andrews, Target-motion-induced radar imaging. IEEE Trans. Aerosp. Electron. Syst. AES-16(1), 2-14 (1980)

2. M. Xing, R. Wu, Z. Bao, High resolution ISAR imaging of high speed moving targets. IEE Proc.-Radar Sonar Navig. 152(2), 58-67 (2005)

3. R. Vehmas, J. Jylhä, M. Väilä, et al, Data-driven motion compensation techniques for noncooperative ISAR imaging. IEEE Trans. Aerosp. Electron. Syst. 54(1), 295-314 (2017)

4. F. Colone, D. Pastina, V. Marongiu, VHF cross-range profiling of aerial targets via passive ISAR: signal processing schemes and experimental results. IEEE Trans. Aerosp. Electron. Syst. 53(1), 218-235 (2017)

5. A. Manno-Kovacs, E. Giusti, F. Berizzi, et al, in 2018 IEEE Radar Conference (RadarConf18). Automatic target classification in passive ISAR range-crossrange images (IEEE, Oklahoma, USA, 2018), pp. 0206-0211

6. J. M. Munoz-Ferreras, F. Perez-Martinez, in 2007 IET International Conference on Radar Systems. Extended envelope correlation for range bin alignment in ISAR (IET, Edinburgh, UK, 2007), pp. 1-5. https://doi.org/10. 1049/cp:20070515

7. D. Zhu, L. Wang, Y. Yu, et al, Robust ISAR range alignment via minimizing the entropy of the average range profile. IEEE Geosci. Remote Sens. Lett. 6(2), 204-208 (2009)

8. J. M. Muñoz-Ferreras, F. Pérez-Martínez, Subinteger range-bin alignment method for ISAR imaging of noncooperative targets. EURASIP J. Adv. Sig. Process. 2010, 1-16 (2010)

9. J. Xu, T. Su, K. Wu, in 2016 CIE International Conference on Radar (RADAR). GPU accelation of parallel range alignment in ISAR real-time imaging (IEEE, Guangzhou, China, 2016), pp. 1-4. https://doi.org/10.1109/RADAR. 2016.8059449

10. L. Xi, L. Guosui, J. Ni, Autofocusing of ISAR images based on entropy minimization. IEEE Trans. Aerosp. Electron. Syst. 35(4), 1240-1252 (1999)

11. J. Xue, Q. Zhang, Y. Wang, et al, Fast estimation of high-order motion parameters for real-time ISAR imaging. IET Radar Sonar Navig. 13(12), 2244-2254 (2019)

12. M. Prickett, C. Chen, in EASCON'80; Electronics and Aerospace Systems Conference. Principles of inverse synthetic aperture radar/ISAR/imaging (IEEE, Arlington, USA, 1980), pp. 340-345

13. T. Itoh, H. Sueda, Y. Watanabe, Motion compensation for ISAR via centroid tracking. IEEE Trans. Aerosp. Electron. Syst. 32(3), 1191-1197 (1996)

14. J. Wang, D. Kasilingam, Global range alignment for ISAR. IEEE Trans. Aerosp. Electron. Syst. 39(1), 351-357 (2003)

15. J. Wang, X. Liu, Improved global range alignment for ISAR. IEEE Trans. Aerosp. Electron. Syst. 43(3), 1070-1075 (2007)

16. M. Martorella, F. Berizzi, B. Haywood, Contrast maximisation based technique for 2-d ISAR autofocusing. IEE Proc.-Radar Sonar Navig. 152(4), 253-262 (2005)

17. F. Berizzi, G. Corsini, Autofocusing of inverse synthetic aperture radar images using contrast optimization. IEEE Trans. Aerosp. Electron. Syst. 32(3), 1185-1191 (1996)

18. L. Zhang, J.-I. Sheng, J. Duan, et al, Translational motion compensation for ISAR imaging under low SNR by minimum entropy. EURASIP J. Adv. Sig. Process. 2013(1), 33 (2013)

19. L. Liu, F. Zhou, M. Tao, et al, Adaptive translational motion compensation method for ISAR imaging under low SNR based on particle swarm optimization. IEEE J. Sel. Top. Appl. Earth Obs. Remote. Sens. 8(11), 5146-5157 (2015)

20. J. Wang, L. Zhang, L. Du, et al, Noise-robust motion compensation for aerial maneuvering target ISAR imaging by parametric minimum entropy optimization. IEEE Trans. Geosci. Remote Sens. 57(7), 4202-4217 (2019)

21. S. J. Wright, Coordinate descent algorithms. Math. Program. 151(1), 3-34 (2015)

22. Y. Xu, W. Yin, A block coordinate descent method for regularized multiconvex optimization with applications to nonnegative tensor factorization and completion. SIAM J. Imaging Sci. 6(3), 1758-1789 (2013)

23. G. Y. Delisle, H. Wu, Moving target imaging and trajectory computation using ISAR. IEEE Trans. Aerosp. Electron. Syst. 30(3), 887-899 (1994)

24. S. Zhang, Y. Liu, X. Li, Fast entropy minimization based autofocusing technique for ISAR imaging. IEEE Trans. Sig. Process. 63(13), 3425-3434 (2015)

25. H.-J. M. Shi, S. Tu, Y. Xu, et al, A primer on coordinate descent algorithms. arXiv preprint arXiv:1610.00040 (2016). https://arxiv.org/abs/1610.00040 
26. M. Lourakis, A. A. Argyros, in Tenth IEEE International Conference on Computer Vision (ICCV'05) Volume 1. Is Levenberg-Marquardt the most efficient optimization algorithm for implementing bundle adjustment?, vol. 2 (IEEE, Beijing, China, 2005), pp. 1526-1531

27. W. S. Cleveland, Robust locally weighted regression and smoothing scatterplots. J. Am. Stat. Assoc. 74(368), 829-836 (1979)

28. J. Wang, X. Liu, Sar minimum-entropy autofocus using an adaptive-order polynomial model. IEEE Geosci. Remote Sens. Lett. 3(4), 512-516 (2006)

29. Q. Dong, L. Zhang, G. Xu, et al, Envelope alignment algorithm for inverse synthetic aperture radar imaging based on splitting sub-apertures. J. Xi'an Jiaotong Univ. 48(12), 107-139 (2014)

30. W. S. Cleveland, S. J. Devlin, E. Grosse, Regression by local fitting: methods, properties, and computational algorithms. J. Econ. 37(1), 87-114 (1988)

31. T. J. Kragh, A. A. Kharbouch, in 2006 International Conference on Image Processing. Monotonic iterative algorithms for SAR image restoration (IEEE, Atlanta, USA, 2006), pp. 645-648

\section{Publisher's Note}

Springer Nature remains neutral with regard to jurisdictional claims in published maps and institutional affiliations.

\section{Submit your manuscript to a SpringerOpen ${ }^{\circ}$ journal and benefit from:}

- Convenient online submission

- Rigorous peer review

- Open access: articles freely available online

- High visibility within the field

- Retaining the copyright to your article

Submit your next manuscript at $\gg$ springeropen.com 